\title{
Modifiable parenting factors associated with adolescent alcohol misuse: $A$ systematic review and meta-analysis of longitudinal studies
}

\author{
Marie B H Yap ${ }^{1,2 \S}$, Tony W K Cheong ${ }^{3}$, Foivos Zaravinos-Tsakos ${ }^{3}$, Dan I Lubman ${ }^{4}$, \\ Anthony F Jorm ${ }^{2}$
}

\author{
${ }^{1}$ School of Psychological Sciences, Monash Institute of Cognitive and Clinical \\ Neurosciences, Monash University, 18 Innovation Walk, Clayton, Victoria 3800, Australia \\ ${ }^{2}$ School of Population and Global Health, University of Melbourne, 207 Bouverie Street, \\ Carlton 3053, Australia
}

${ }^{3}$ Vrije Universiteit Amsterdam, Section of Clinical Psychology, Faculty of Behavioural and Movement Sciences, Amsterdam, Noord-Holland, NL

${ }^{4}$ Turning Point, Eastern Health and Eastern Health Clinical School, Monash University, 54

Gertrude Street, Fitzroy, Victoria 3065, Australia

${ }^{\S}$ Corresponding author: Dr Marie Yap, marie.yap@ monash.edu

Running head: Parenting and adolescent alcohol misuse

Word count: 9936

Conflict of interest declaration: Dan Lubman has provided consultancy advice to Lundbeck, and has received travel support and speaker honoraria from Astra Zeneca, Bristol Myers Squibb, Janssen and Lundbeck. All other authors declare no conflict of interest.

Review Protocol Registration number: CRD42015016723 (PROSPERO). Available from http://www.crd.york.ac.uk/PROSPERO/display_record.asp?ID=CRD42015016723 


\section{Abstract}

Aims

Adolescent alcohol misuse is a growing global health concern. Substantial research suggests that parents have an important role in reducing young people's risk for early initiation and alcoholrelated harms. To facilitate the translation of the extensive evidence base into preventive resources for parents, we conducted a systematic review and meta-analyses of longitudinal studies examining the range of modifiable parenting factors that are associated with adolescent alcohol initiation and levels of later use/misuse.

\section{Methods}

A systematic literature search was conducted in PubMed, PsycINFO, and Embase. Studies were included if they (i) used a longitudinal design; (ii) were published in English; (iii) measured any modifiable parenting factors in adolescence as predictors; (iv) assessed any alcohol-related outcome variables in adolescence, and/or alcohol-related problems in adulthood; and (v) had a follow-up interval of at least one year. Parental behaviors were categorized into twelve parenting factors. Stouffer's $p$ analyses were used to determine whether the associations between variables were reliable; when there were sufficient studies available, meta-analyses were also conducted to estimate mean effect sizes.

\section{Results}

Based on 131 studies, three risk factors (parental provision of alcohol, favorable parental attitudes towards alcohol, and parental drinking) and four protective factors (parental monitoring, parentchild relationship quality, parental support, and parental involvement) were identified as longitudinal predictors of both alcohol initiation and levels of later alcohol use/misuse, based on their significant results in both Stouffer's $p$ analyses and meta-analyses. The mean effect sizes were mostly small ( $r s=-0.224$ to 0.263 ).

\section{Conclusions}

Parenting interventions to prevent adolescent alcohol misuse should be designed to reduce parental provision of alcohol, favorable parental attitudes towards alcohol, and parental drinking; and increase parental monitoring, parent-child relationship quality, parental support, and parental involvement. The small mean effect sizes indicate the need for comprehensive approaches that target parental factors as one component of multi-component interventions. 


\section{Background}

Alcohol misuse in young people is a growing global health priority [1], with the World Health Organisation's global strategy to reduce the harmful use of alcohol including a specific focus on young people [2]. Underage drinking has wide-ranging and enduring adverse effects, with the strongest evidence indicating a likely causal link to subsequent substance dependence, physical harms (fatal overdose, road traffic accidents and other accidental injuries), and cognitive impairment [3]. Of particular concern is the impact of alcohol use during the adolescent developmental phase, which is a key period of transition marked by significant neurodevelopment, with regards to young people's social, cognitive and emotional development [2, 3]. Given that adolescence is also the developmental period when alcohol use typically begins [2], with adolescent patterns of drinking related to later alcohol use disorders [4-6], it marks an opportune time for prevention.

The call for prevention of underage drinking has been made and echoed in many countries, including the United States of America [7], the United Kingdom (UK, [8]), and Australia [9]. In particular, national guidelines were published in both the UK [8] and Australia [9] in 2009 which recommend that adolescents under the age of 18 should delay initiating alcohol consumption for as long as possible, and that those below 15 years not drink any alcohol at all (henceforth any drinking before age 15 is considered alcohol misuse). Of note, both sets of guidelines are directed at parents and carers (henceforth 'parents') of young people as implementers of the recommendations against underage drinking; hence identifying parents as key players in the prevention of adolescent alcohol misuse.

Consistent with this call, the recent systematic review of reviews by Stockings and colleagues [10] highlights the potential of adolescent alcohol use prevention interventions delivered to the family or parents, especially interventions that focus on building parenting skills and parent-child relationships [11]. Of particular note is that aside from taxation initiatives, which have a medium-to-large effect on reducing alcohol consumption and problematic use in young people, prevention interventions with parents of young people which deliver skills training and cognitive behavioural therapy in 
addition to education about alcohol use emerged as the only set of psychologically-based prevention interventions with at least a small effect size. However, as evident from the Cochrane review of universal family-based prevention interventions [11], whilst most of the interventions were designed to target some parenting risk and protective factors for adolescent alcohol misuse, 10 of the 12 included studies intervened directly with both parent and child. Hence it is unclear how much of the intervention effects could be attributed to change in parenting factors. There was also variation in the number and type of parenting factors targeted across the interventions, ranging from a singlefactor focus (e.g. alcohol-specific communication [12]) to a more integrative approach of targeting multiple parent and child factors (e.g. lowa Strengthening Families Program and Preparing for the Drug-Free Years Program [13], where adolescents are taught refusal skills, in addition to parents being taught skills in discipline/rules, parent-child communication, bonding and conflict management). Moreover, although most programs target more than one parenting factor, the translation of the research evidence into parent skills training interventions is still lagging behind the rapidly growing evidence base, with no existing program to date adequately addressing the wide range of parenting factors supported by evidence as predicting adolescent alcohol misuse [14].

To better facilitate research translation, we need to identify more clearly the factors that are supported by a sound evidence base. This is particularly important in communities where alcohol use is such an accepted norm, especially amongst young people, such that prevention or disapproval of alcohol use runs counter to beliefs of young people who view alcohol use as a positive experience with minimal negative consequences [15]. Moreover, parents continue to receive inconsistent messages about what they should do to combat the concerning problem of alcohol misuse and its harms in young people, from the media, anecdotal experience (including cultural norms, family background, etc.), and even research evidence $[16,17]$.

There are two key challenges to successful translation of research evidence. The first is the methodological quality of research studies, which have largely utilized study-specific measures that 
are inconsistently defined across studies and have varying psychometric properties; and reported findings with varying levels of adequacy for the purpose of a quantitative synthesis [14]. Consequently, the only existing syntheses to date of parenting factors associated with adolescent alcohol use have been narrative systematic reviews [14, 17-19]. Most of these reviews partly compensate for the inability to conduct a meta-analysis by using various methods of synthesizing the findings and assessing the quality of the evidence $[14,18,19]$. Nonetheless, descriptive qualitative syntheses cannot objectively quantify the effect sizes of associations between parenting factors and adolescent alcohol use. A systematic review that incorporates meta-analytic techniques is required to facilitate the drawing of firmer conclusions and hence substantiate recommendations to parents about what they should do to prevent adolescent alcohol misuse.

Another challenge to research translation is that extant reviews focus on a specific parenting factor or a small subset of parenting factors [17-20]. While a narrower focus allows more detailed discussion about the target parenting factor(s), it is important to consider the wide range of parenting factors that increase or decrease risk for adolescent alcohol use. While the 2010 systematic review by Ryan and colleagues [14] attempted to do this, the conclusions of that review were limited by the absence of meta-analytic findings.

The overall aim of the current review is to address both above-mentioned challenges to research translation by systematically synthesizing the evidence from longitudinal studies about the range of risk and protective factors for adolescent alcohol use that parents can potentially influence or modify. Specifically, this review will update Ryan and colleagues' review [14], use a more comprehensive search strategy than that employed in the earlier review, as well as strengthen the conclusions of the review by conducting meta-analyses of primary studies, where possible, for each of the 12 parenting factors and two adolescent alcohol use outcomes (initiation and levels of later use/misuse) examined. The 12 parenting factors are: (1) provision of alcohol, (2) parental monitoring, (3) favorable attitudes towards alcohol, (4) parental alcohol use, (5) parent-child relationship quality, 
(6) parental support, (7) parental involvement, (8) rules about alcohol use, (9) family conflict, (10) parental discipline, (11) alcohol-specific communication, and (12) general communication. 


\section{Method}

The protocol of the current systematic review was registered on PROSPERO (registration

number: CRD42015016723).

\section{Search Strategy}

A systematic literature search was first conducted in three electronic databases (PubMed, PsycINFO, and Embase) on February $5^{\text {th }} 2015$, and updated on June $15^{\text {th }} 2016$. Appendix A presents the search strings used in each database. We developed these search strings by combining terms indicative of parenting, alcohol, and adolescence/adolescents. Both index terms (i.e., MeSH terms, Thesaurus, and Emtree terms) and text words were employed. Searches were restricted to peerreviewed articles written in English. No restriction on publication date was imposed. Additional articles were identified by checking reference lists of the included studies, and by scrutinizing articles included in the four recent systematic reviews [14, 17-19].

\section{Study Selection}

After removal of duplicates, studies were selected in two phases. At the first phase, all titles and abstracts were checked for potential relevance; at the second phase, studies identified as being potentially relevant were re-assessed based on the full-text articles. Studies were included in this review if they: (i) used a longitudinal study design; (ii) were published in English; (iii) contained one or more modifiable parenting factors measured in adolescence or pre-adolescence (before age 18) as predictors; (iv) contained one or more outcome variables defined as any alcohol drinking patterns and/or alcohol-related problems in adolescence (between ages 12 and <18), and/or any alcoholrelated problems during adulthood (from age 18 onwards); and ( $v$ ) the outcome variables were assessed at least one year after the initial parenting factor was measured (since a follow-up interval of shorter than one year was considered insufficient for the inference of temporality).

Studies were excluded if: (i) their main purpose was to evaluate an intervention or treatment program; (ii) different parenting factors were combined as a single predictor; or (iii) the 
alcohol-related outcomes were combined with other substance use or externalizing behaviors as a composite measure.

\section{Data Extraction}

A pilot-tested spreadsheet was used for data collection. Extracted information included source of participants (general population sample vs. clinical/minority sample), country, sample size, baseline mean age, follow-up time interval, details of parenting factor (independent variable) and alcohol-related outcome (dependent variable), $p$ value, as well as the magnitude and direction of effect. In this review, the unit of analysis was the association between a specific parenting factor and an alcohol-related outcome. $p$ values and effect sizes were extracted to quantify these associations. To standardize the data selection process, a set of decision rules were developed for studies reporting multiple associations between the same relevant variables, and for studies involving duplicate data. Appendix B is the codebook used for data extraction and analyses, which contains all pre-specified rules. When a study reported analyses across multiple time points, we extracted the associations involving the longest and the shortest time intervals. In terms of parenting variables, we collected data from studies where maternal and paternal behaviors had been analyzed separately, as well as from studies where the two parents' behaviors had been treated as a composite measure. Where possible, gender-specific associations were also extracted. Two authors (W.C. and F.Z.T.) independently extracted data from all the included studies. In case of issues not directly solvable through discussions, a decision was made via consultation with A.J. or M.Y.

\section{Categorizations of Parenting Factors and Alcohol-related Outcomes}

Parenting factors were defined as any family variables that are potentially modifiable by parents. Thus, factors that are not readily modifiable by parents at the individual level (e.g., family income, parental education, and mental health problems/disorders) were not examined in this review. Due to the diversity in the way parenting factors were named, defined, and measured in the literature, they had to be categorized into different themes before any meaningful quantitative syntheses could be conducted. Categorization was based on the twelve factors identified in an 
earlier review [14], with some definitions slightly modified on the account of newly included studies (see Table 1).

\section{[Insert Table 1 here]}

Based on the outcome measures of the original studies, alcohol-related variables were also divided into two groups and analyzed separately. Studies were included in the analyses of alcohol initiation if they measured onset of alcohol consumption among baseline abstainers of any age (henceforth referred to as 'alcohol onset'). Alcohol initiation also included any alcohol-related outcomes (e.g. excessive/binge drinking; frequency/quantity) before age 15, since the Australian and UK guidelines both recommend that adolescents under age 15 refrain from any alcohol use $[8,9]$; hence, any alcohol-related outcomes before this age represent early initiation and are considered harmful (henceforth referred to as 'levels of early alcohol use'). Studies were included in the analyses for levels of later alcohol use/misuse if they investigated frequency/quantity of drinking or any alcohol-related problems (e.g. frequency of drunkenness, excessive/binge drinking, abuse/dependence) from age 15 onwards. Table 1 presents the hypothesized associations between each specific parenting factor and the two alcohol-related outcomes.

\section{Risk of bias assessment}

The risk of biases for each individual study was assessed with the checklist derived from Hayden, Cote, and Bombardier [21]. This comprehensive checklist is applied when assessing potential biases in non-randomized studies, and was used in the previous systematic review by Visser and colleagues [19]. Six domains of biases are included in the checklist: (1) study participation, (2) study attrition, (3) predictor measurement, (4) outcome measurement, (5) confounding measurement, and (6) analysis. For the present review, only five domains of biases, encompassing 13 criteria, were considered relevant and selected (see Table 2). Each criterion was rated as + (the criterion was met), \pm (the criterion was partly met), - (the criterion was not met), or ? (unsure); accordingly, a score of 2, 1, 0 , or 0 was given to the criterion. When a criterion was considered not applicable for the study, it was 
rated as NA. For each particular domain of bias, if $\leq 50 \%$ of the maximum score (excluding any nonapplicable criteria) was obtained, a score of 1 was added to the number of biases for the study. Hence, for each individual study, the total number of biases could range from zero to five. Two authors (W.C. and F.Z.T.) independently assessed the risk of biases for all the included studies. All disagreements were resolved through discussions.

[Insert Table 2 here]

\section{Data Analyses}

Due to the diversity of methodologies and statistical approaches, standard effect size measures could not be extracted from all the included studies. Hence, only a subset of studies could be included in meta-analyses. Using the analytic approach of various recent systematic reviews [2224], Stouffer's method of combining $p$ values [25] was employed to synthesize results from all the studies; findings were then supplemented by meta-analyses of a subset of studies.

Studies were included in meta-analyses if they: (i) reported a correlation coefficient $r$, or an alternative effect size measure (e.g., odds ratio with confidence interval) that could be converted to $r$ using the software Comprehensive Meta-Analysis (CMA [26]); and (ii) this effect size was not adjusted for any covariates. Data from clinical/minority samples were excluded from meta-analyses because the current review focused on factors that may facilitate prevention in the community more broadly. $r$ was selected as the measure of effect size because it was the most commonly reported. Since the number and nature of controlled covariates were highly heterogeneous among the included studies (e.g., gender, ethnicity, family history, and other baseline characteristics), only unadjusted effect sizes were meta-analyzed in order to maintain the similarities across data from different studies.

Since a study could report analyses involving mothers and/or fathers separately, and/or for both parents together, to avoid data dependency, a decision hierarchy was pre-specified to ensure only one association from each study was included in the relevant Stouffer's $p$ analysis and main 
meta-analysis. The hierarchy specifies the following in descending order of preference: (i) composite parental behaviors, (ii) maternal behaviors, and (iii) paternal behaviors. When there were sufficient studies available (i.e., at least two independent studies), supplementary meta-analyses were performed to explore the mean effect sizes for each parent. Supplementary meta-analyses are mentioned below only if they were conducted.

Stouffer's $p$ analyses. By testing the combined significance levels rather than estimating mean effect sizes, Stouffer's $p$ values determined whether the associations between each parenting factor and alcohol-related outcomes were reliable. To test the directional hypotheses, $p$ values from all the included studies were first converted to one-tailed values. A Stouffer's $z$ was calculated by dividing the sum of $z\left(p_{i}\right)$ values by the square root of $k$, with $z\left(p_{i}\right)$ representing the $z$ values for each extracted association and $k$ representing the number of associations involved in each analysis. When the resulting Stouffer's $z$ corresponded to a $p$ value lower than 0.05 , the null hypothesis of no association was rejected.

Meta-analytic procedures. A meta-analysis was conducted when there were at least two independent studies available. Due to the differences in settings and measures among the included studies, considerable heterogeneity was expected a priori. Hence, a random effects model was used in all analyses. The resulting mean effect sizes (i.e., $r$ s) were interpreted based on Cohen's guidelines[27], with $0.10,0.30$, and 0.50 indicating small, medium, and large effects respectively. To test and quantify heterogeneity, $Q$ and $l^{2}$ statistics were calculated. A significant $Q$ rejects the null hypothesis of homogeneity, and indicates the presence of between-study variation. $I^{2}$ gives the percentage of variation that could be explained by between-study heterogeneity rather than by sampling error, with $25 \%, 50 \%$, and $75 \%$ indicating low, moderate, and high heterogeneity respectively[28]. All meta-analyses were performed using CMA Version 3.3.070 [26].

Publication bias. When a meta-analysis involved three or more independent studies, publication bias was checked by visual inspection of the funnel plot, and by using Egger's regression 
asymmetry test [29]. Egger's test quantifies the asymmetry captured by the funnel plot and determines whether it is significant. When asymmetry was detected, Duval and Tweedie's nonparametric trim-and-fill procedure [30] was used to impute the potentially missing studies, and recompute a mean effect size. Tests for publication bias are mentioned below only if they were conducted (i.e., only if there were at least three independent studies available).

Moderator analyses. Moderator effects were tested when there were at least four associations available (as required by CMA [26]). Univariate meta-regression analyses were performed for continuous moderators: baseline age and follow-up time interval. The resulting slopes represented the changes in $r$ s given a unit of change in each moderator. Subgroup analyses were performed for categorical moderators: gender of adolescents $(1=$ male sample, $2=$ female sample, 3 = combined sample) and outcome measure (for analyses of initiation: 1 = alcohol onset, 2 = levels of early alcohol use; for analyses of later use/misuse: 1 = frequency/quantity, 2 = alcohol-related problems). In these analyses, associations within each subgroup were pooled separately, and the between-group differences were tested.

Sensitivity analyses. When a study reported analyses across multiple time points, a decision was made to include the association with the longest time interval in the meta-analysis (see Appendix B). However, sensitivity analyses using the shortest time intervals were also conducted to examine the potential influences on pooled effect sizes.

Interpretations of findings. When interpreting the findings of the current review, we categorized parenting factors into three groups based on their results in both Stouffer's $p$ analyses and meta-analyses: factors with a sound, convergent evidence base (i.e., longitudinal predictors of both alcohol initiation and levels of later alcohol use/misuse, based on their significant results in both analytic approaches); factors with emerging evidence (i.e., significant predictors of at least one alcohol-related outcome, based on their consistent findings in the two analytic approaches); and 
factors showing weak evidence (i.e., parenting factors that only showed inconsistent results between the two analytic approaches, or yielded non-significant findings in both approaches).

\section{Results}

Figure 1 illustrates the entire process of systematic literature search. After removal of duplicates, 4705 titles and abstracts were screened, of which 850 articles were assessed for eligibility based on full texts. 131 studies, which encompassed 416 associations, were identified as being eligible for inclusion. These studies were published between 1986 and 2016, with the majority conducted in North America $(n=92)$, and the remainder in Europe $(n=31)$, Australia $(n=5)$, New Zealand $(n=1)$, and both Australia and the United States $(n=2)$. For the Stouffer's $p$ analyses, 181 associations from 60 studies were included in the analyses of alcohol initiation, and 235 associations from 84 studies were included in the analyses for levels of later alcohol use/misuse. Only 165 associations from 48 studies were included in meta-analyses. Appendix C presents the detailed characteristics and $p$ values for each study, with reasons provided for studies not eligible for metaanalyses.

\section{[Insert Figure 1 here]}

\section{Risk of bias assessment}

Of the 131 studies included in this review, 79 studies (60\%) did not show biases in any domains. Of the remainder, 35 studies had biases in only one domain; 13 studies had biases in two domains; three studies had biases in three domains; and only one study had biases in four domains (see Appendix D). Among the five specific domains, the most commonly observed one was study attrition biases, which were shown in 42 studies ( $81 \%$ of the studies that had biases in at least one domain). Of the other domains, predictor measurement biases were shown in eleven studies; biases in analysis were shown in eight studies; study participation biases were shown in seven studies; and outcome measurement biases were shown in six studies. 
Findings from Stouffer's $p$ and meta-analyses are summarized in text below, divided into alcohol-specific parenting factors and other general parenting factors and ordered by alphabetical order. Sensitivity analyses using the shortest time intervals have not been reported because their impacts on findings were considered negligible, with changes in $r$ s ranging from -0.004 to 0.02 . Tables 3 and 4 present the results of the main and supplementary analyses respectively. The pooled effect sizes from the main analyses are shown in Figures $2 a$ and $2 b$ (see Appendix $E$ for the individual forest plots of each main analysis). Results of meta-regression and subgroup analyses are shown in Appendices F and $G$. The complete lists of included studies and excluded studies (with reasons for exclusion) are found in Appendices $\mathrm{H}$ and I respectively. The PRISMA (Preferred Reporting Items for Systematic Reviews and Meta-Analyses) checklist, on which the reporting of the current review was based, is found in Appendix J.

[Insert Table 3 here]

[Insert Table 4 here]

\section{Alcohol-specific Parenting Factors}

Alcohol-specific communication. Only two associations, derived from two independent studies, were meta-analyzed for the prospective link between alcohol-specific communication and delayed alcohol initiation. A non-significant mean effect size emerged $(r=0.124,95 \% \mathrm{Cl}[-0.137$, $0.369], p=0.352)$, in the opposite direction to that hypothesized, with high heterogeneity $\left(l^{2}=95 \%\right)$. Stouffer's $p$ analysis also yielded a non-significant result $(p=0.207)$. However, forest plot inspection revealed that there was no overlap between the two studies involved. Whilst one study measuring general discussions about alcohol reported a non-significant result $(r=-0.002,95 \% \mathrm{Cl}[-0.017,0.012]$, $p=0.733 ;[31])$, the other study measuring parental disclosure of negative alcohol experiences showed a significant, almost-medium effect size in the opposite direction to that hypothesized ( $r=$ $0.260,95 \% \mathrm{Cl}[0.145,0.368], p<0.001 ;[32])$. Supplementary meta-analyses revealed that neither mother-child nor father-child communication was significantly related to alcohol initiation. 
There were insufficient studies to conduct a meta-analysis for the association between alcohol-specific communication and reduced levels of alcohol use/misuse. However, a nonsignificant Stouffer's $p$ was found $(p=0.259)$.

Favorable attitudes toward alcohol use. Based on five associations from five studies, favorable parental attitudes toward alcohol use was related to early alcohol initiation in adolescents, with a small but significant pooled effect size $(r=0.114,95 \% \mathrm{Cl}[0.033,0.193], p=0.006)$, and high heterogeneity $\left(I^{2}=79 \%\right)$. A significant Stouffer's $p$ was also found $(p<0.0001)$. The funnel plot and Egger's test revealed that publication bias might exist ( $p=0.039$, one-tailed). The trim-and-fill procedure indicated that two studies were potentially missing, but the pooled effect size remained significant after imputation $(r=0.073,95 \% \mathrm{Cl}[0.003,0.143], p=0.043)$. Meta-regression and subgroup analyses revealed that follow-up time interval $(p=0.006)$ and outcome measure $(p<0.001)$ were significant moderators, with smaller effect sizes found in studies involving a longer time interval and studies that measured alcohol onset. Finally, supplementary meta-analyses showed that only paternal attitudes was significantly associated with early initiation $(r=0.058,95 \% \mathrm{Cl}[0.015$, $0.101], p=0.008)$.

Based on seven associations from five studies, favorable parental attitudes was related to higher levels of alcohol use/misuse in adolescents, with a small but significant pooled effect size ( $r=$ $0.182,95 \% \mathrm{Cl}[0.139,0.224], p<0.001)$, and moderate-to-high heterogeneity $\left(l^{2}=62 \%\right)$. Stouffer's $p$ analysis also showed a consistent result $(p<0.0001)$. There was no apparent publication bias. Supplementary meta-analyses revealed that both maternal attitudes $(r=0.175,95 \% \mathrm{Cl}[0.134$, $0.215], p<0.001)$ and paternal attitudes $(r=0.167,95 \% \mathrm{Cl}[0.125,0.209], p<0.001)$ were positively associated with levels of alcohol use/misuse.

Parental alcohol use. Based on ten associations from ten independent studies, parental alcohol use was positively associated with early alcohol initiation in adolescents, with a small but significant pooled effect size $(r=0.116,95 \% \mathrm{Cl}[0.049,0.182], p=0.001)$, and high heterogeneity $\left(I^{2}\right.$ 
$=93 \%)$. Stouffer's $p$ analysis also yielded a significant result $(p<0.0001)$. The funnel plot and Egger's test revealed that publication bias might exist ( $p=0.001$, one-tailed), but the trim-and-fill approach indicated that no study was missing. Supplementary meta-analyses showed that only paternal drinking was significantly related to early alcohol initiation $(r=0.114,95 \% \mathrm{Cl}[0.005,0.220], p=$ 0.040).

Twelve associations from nine studies were identified for the prospective link between higher levels of parental alcohol use and higher levels of alcohol use/misuse in adolescents, yielding a small but significant mean effect size $(r=0.153,95 \% \mathrm{Cl}[0.088,0.216], p<0.001)$ with high heterogeneity $\left(I^{2}=83 \%\right)$. A significant Stouffer's $p$ was also found $(p<0.0001)$. There was no evidence of publication bias. Supplementary meta-analyses revealed that both maternal drinking $(r=$ $0.159,95 \% \mathrm{Cl}[0.101,0.216], p<0.001)$ and paternal drinking $(r=0.158,95 \% \mathrm{Cl}[0.085,0.231], p<$ 0.001) were positively associated with adolescent use/misuse.

Provision of alcohol. Only two associations, extracted from two independent studies, were meta-analyzed for the positive, prospective link between parental provision of alcohol and early alcohol initiation. A small but significant mean effect size emerged $(r=0.205,95 \% \mathrm{Cl}[0.162,0.248]$, $p<0.001)$, with zero heterogeneity $\left(I^{2}=0 \%\right)$. Stouffer's $p$ analysis also yielded a significant result $(p<$ $0.0001)$.

Four associations from two studies were meta-analyzed for the positive, longitudinal link between provision of alcohol and levels of alcohol use/misuse, yielding a significant, almost-medium pooled effect size $(r=0.263,95 \% \mathrm{Cl}[0.210,0.314], p<0.001)$ with moderate heterogeneity $\left(I^{2}=\right.$ 54\%). Stouffer's $p$ analysis also showed a consistent finding $(p<0.0001)$.

Rules about alcohol use. Only two associations, extracted from two independent studies, were meta-analyzed for the prospective link between having parental rules about alcohol use and delayed alcohol initiation. A small but significant mean effect size emerged $(r=-0.195,95 \% \mathrm{Cl}[-$ 
$0.337,-0.044], p=0.012)$, with moderate-to-high heterogeneity $\left(I^{2}=67 \%\right)$. Stouffer's $p$ analysis also yielded a significant result $(p=0.0001)$.

There were insufficient studies to conduct a meta-analysis for the association between having rules about alcohol use and reduced levels of alcohol use/misuse. However, a significant Stouffer's $p$ was found $(p<0.0001)$.

\section{General Parenting Factors}

Family conflict. Based on eight associations from seven studies, higher levels of family conflict were related to early alcohol initiation, with a small but significant mean effect size $(r=$ $0.108,95 \% \mathrm{Cl}[0.050,0.166], p<0.001)$, and high heterogeneity $\left(I^{2}=91 \%\right)$. A significant Stouffer's $p$ was also found $(p<0.0001)$. The funnel plot and Egger's test revealed that publication bias might exist ( $p=0.001$, one-tailed). The trim-and-fill procedure suggested that three studies were potentially missing, but the pooled effect size remained significant after imputation $(r=0.085,95 \%$ $\mathrm{Cl}[0.037,0.132], p<0.001)$. Meta-regression analyses revealed that baseline age $(p<0.001)$ and follow-up time interval $(p<0.001)$ were significant moderators, with smaller effect sizes found in studies with a younger baseline age and studies involving a longer time interval.

Three associations from three studies were identified for the longitudinal link between higher levels of family conflict and higher levels of alcohol use/misuse, yielding a small, nonsignificant mean effect $(r=0.112,95 \% \mathrm{Cl}[-0.026,0.245], p=0.111)$ with high heterogeneity $\left(I^{2}=\right.$ 81\%). However, a significant Stouffer's $p$ was found $(p<0.0001)$. There was no evidence of publication bias.

General communication. Only two associations from two studies were meta-analyzed for the longitudinal link between general parent-child communication and delayed alcohol initiation, yielding a non-significant mean effect size $(r=-0.033,95 \% \mathrm{Cl}[-0.198,0.133], p=0.696)$ with high heterogeneity $\left(I^{2}=91 \%\right)$. However, a significant result was found in Stouffer's $p$ analysis $(p<0.0001)$. Forest plot inspection revealed that there was no overlap between the two studies involved in meta- 
analysis. Whilst a small but significant effect size was found in one study that measured adolescent active disclosure of daily activities to parents $(r=-0.120,95 \% \mathrm{Cl}[-0.198,-0.041], p=0.003 ;[33])$, a non-significant effect in the opposite direction was observed in the other study that measured parent-child verbal communication in general $(r=0.050,95 \% \mathrm{Cl}[-0.011,0.111], p=0.109 ;[34])$.

Four associations from three studies were meta-analyzed for the prospective link between general communication and reduced levels of alcohol use/misuse, yielding a non-significant mean effect size $(r=-0.029,95 \% \mathrm{Cl}[-0.133,0.077], p=0.597)$ with high heterogeneity $\left(I^{2}=91 \%\right)$. A nonsignificant Stouffer's $p$ was also found $(p=0.085)$. There was no apparent publication bias. However, forest plot inspection revealed that one study reported a significant result in the opposite direction to that hypothesized $(r=0.080,95 \% \mathrm{Cl}[0.040,0.120], p<0.001 ;[35])$. This study measured general discussions about daily issues, whereas the other two studies assessed positive aspects of parentchild communication. After post-hoc removal of the former study, the effect size became significant $(r=-0.080,95 \% \mathrm{Cl}[-0.117,-0.042], p<0.001)$, and the heterogeneity decreased substantially $\left(I^{2}=\right.$ $0 \%$ ). Since the main meta-analysis only involved measures of mother-child communication from the three studies (see Appendix B for the details of decision hierarchy), supplementary meta-analysis for mothers showed the identical result. Analysis for fathers involved measures of father-child communication from the same studies, yielding a non-significant mean effect size. However, after post-hoc removal of the study by Cookston and Finlay [35], a significant, small effect size emerged ( $r$ $=-0.080,95 \% \mathrm{Cl}[-0.118,-0.042], p<0.001)$, with no apparent heterogeneity $\left(I^{2}=0 \%\right)$.

Parent-child relationship quality. Based on six associations from five studies, better parent-child relationship quality was associated with delayed alcohol initiation, with a small but significant pooled effect size $(r=-0.106,95 \% \mathrm{Cl}[-0.190,-0.020], p=0.016)$, and high heterogeneity $\left(I^{2}=96 \%\right)$. Stouffer's $p$ analysis also yielded a significant result $(p<0.0001)$. The funnel plot and Egger's test suggested that publication bias might exist ( $p=0.042$, one-tailed), but the trim-and-fill approach indicated that no study was missing. Meta-regression and subgroup analyses revealed that 
baseline age $(p<0.001)$, follow-up time interval $(p=0.041)$, gender $(p<0.001)$, and outcome measure $(p=0.015)$ were significant moderators, with smaller effect sizes found in studies with a younger baseline age, studies with a longer time interval, studies involving a combined-gender sample, and studies that measured alcohol onset. When examined separately in supplementary meta-analyses, neither mother-child nor father-child relationship quality was significantly associated with delayed alcohol initiation.

Eight associations from six studies were identified for the longitudinal link between better parent-child relationship quality and reduced levels of alcohol use/misuse, yielding a small but significant pooled effect size $(r=-0.119,95 \% \mathrm{Cl}[-0.156,-0.081], p<0.001)$ with high heterogeneity $\left(I^{2}=81 \%\right)$. Stouffer's $p$ analysis also yielded a significant result $(p<0.0001)$. There was no evidence of publication bias. Meta-regression and subgroup analyses revealed that follow-up time interval ( $p$ $<0.001)$, gender $(p<0.001)$, and outcome measure $(p<0.001)$ were significant moderators, with smaller effect sizes found in studies involving a longer time interval, studies reporting genderspecific associations, and studies that measured alcohol-related problems. Since one study involved an exceptionally long time interval (26 years; [36]), a post-hoc sensitivity analysis was conducted by removing this study. After removal, the mean effect size remained significant $(r=-0.155,95 \% \mathrm{Cl}[-$ $0.183,-0.127], p<0.001)$, but the heterogeneity decreased substantially $\left(l^{2}=7 \%\right)$, and all moderator effects became non-significant. Finally, supplementary meta-analyses revealed that both motherchild $(r=-0.133,95 \% \mathrm{Cl}[-0.160,-0.105], p<0.001)$ and father-child relationship quality $(r=-0.172$, $95 \% \mathrm{Cl}[-0.199,-0.144], p<0.001)$ were significantly associated with reduced alcohol use/misuse.

Parental discipline. Based on six associations from six studies, appropriate parental discipline was associated with delayed alcohol initiation, with a small but significant pooled effect size $(r=-0.069,95 \% \mathrm{Cl}[-0.134,-0.003], p=0.042)$, and high heterogeneity $\left(I^{2}=74 \%\right)$. A significant Stouffer's $p$ was also found $(p<0.0001)$. There was no apparent publication bias. Supplementary 
meta-analyses revealed that only maternal discipline was significantly associated with delayed alcohol initiation $(r=-0.126,95 \% \mathrm{Cl}[-0.233,-0.016], p=0.025)$.

There were insufficient studies to conduct a meta-analysis for the association between appropriate parental discipline and reduced levels of alcohol use/misuse. However, a significant Stouffer's $p$ was found $(p<0.0001)$.

Parental involvement. Five associations from three studies examined the prospective link between positive parental involvement and delayed alcohol initiation, yielding a small but significant mean effect size $(r=-0.108,95 \% \mathrm{Cl}[-0.159,-0.057], p<0.001)$ with high heterogeneity $\left(I^{2}=78 \%\right) . \mathrm{A}$ significant Stouffer's $p$ was also found $(p<0.0001)$. There was no evidence of publication bias.

Five associations from three studies were meta-analyzed for the longitudinal link between positive parental involvement and reduced levels of alcohol use/misuse. A small but significant pooled effect size was found $(r=-0.073,95 \% \mathrm{Cl}[-0.126,-0.019], p=0.008)$, with high heterogeneity $\left(I^{2}=89 \%\right)$. Stouffer's $p$ analysis also yielded a significant result $(p<0.0001)$. The funnel plot and Egger's test revealed that publication bias might exist ( $p=0.047$, one-tailed). The trim-and-fill procedure suggested that two studies were potentially missing. After imputation, the pooled effect size became non-significant $(r=-0.038,95 \% \mathrm{Cl}[-0.089,0.013], p=0.148)$. Meta-regression analyses revealed that baseline age $(p=0.002)$ and follow-up time interval $(p<0.001)$ were significant moderators, with smaller effect sizes observed in studies with a younger baseline age and studies involving a longer time interval. Since one study involved an exceptionally long time interval (35 years; [36]), a post-hoc sensitivity analysis was conducted by removing this study. After removal, the mean effect size remained significant $(r=-0.129,95 \% \mathrm{Cl}[-0.183,-0.075], p<0.001)$, but the heterogeneity decreased substantially $\left(I^{2}=31 \%\right)$.

Parental monitoring. Seven associations from six studies were identified for the longitudinal link between higher levels of parental monitoring and delayed alcohol initiation, yielding a small but significant mean effect size $(r=-0.159,95 \% \mathrm{Cl}[-0.218,-0.098], p<0.001)$ with high 
heterogeneity $\left(I^{2}=85 \%\right)$. Stouffer's $p$ analysis also yielded a significant result $(p<0.0001)$. There was no apparent publication bias. Subgroup analyses revealed that gender was a significant moderator ( $p$ <0.001), with a significantly larger effect size found in the female subgroup of one study [37], compared to the male subgroup from the same study, and other studies involving a combinedgender sample.

Twelve associations from nine studies were identified for the prospective link between higher levels of parental monitoring and reduced levels of alcohol use/misuse, yielding a small but significant mean effect size $(r=-0.224,95 \% \mathrm{Cl}[-0.267,-0.180], p<0.001)$ with high heterogeneity $\left(I^{2}\right.$ $=83 \%)$. A significant Stouffer's $p$ was also found $(p<0.0001)$. There was no evidence of publication bias.

Parental support. Based on eight associations from seven studies, higher levels of parental support were associated with delayed alcohol initiation, with a small but significant pooled effect size $(r=-0.117,95 \% \mathrm{Cl}[-0.169,-0.065], p<0.001)$, and moderate-to-high heterogeneity $\left(l^{2}=69 \%\right)$. A significant Stouffer's $p$ was also found $(p<0.0001)$. There was no apparent publication bias. Supplementary meta-analyses revealed that only maternal support was significantly related to delayed initiation $(r=-0.163,95 \% \mathrm{Cl}[-0.307,-0.012], p=0.035)$.

Seven associations from seven independent studies were meta-analyzed for the longitudinal link between higher levels of parental support and reduced levels of alcohol use/misuse. A small but significant pooled effect size was found $(r=-0.110,95 \% \mathrm{Cl}[-0.154,-0.065], p<0.001)$, with moderate-to-high heterogeneity $\left(l^{2}=70 \%\right)$. Stouffer's $p$ analysis also yielded a significant result $(p<$ 0.0001). There was no apparent publication bias. Meta-regression analyses revealed that follow-up time interval was a significant moderator $(p=0.017)$, with smaller effect sizes found in studies involving a longer time interval. Supplementary meta-analyses found that both maternal support $(r=$ $-0.147,95 \% \mathrm{Cl}[-0.202,-0.091], p<0.001)$ and paternal support $(r=-0.131,95 \% \mathrm{Cl}[-0.177,-0.084], p$ $<0.001$ ) were significantly associated with reduced alcohol use/misuse. 
[Insert Figure 2a here]

[Insert Figure $2 \mathrm{~b}$ here] 


\section{Discussion}

The current review systematically synthesized the evidence for 12 potentially modifiable parenting factors as prospective predictors of two adolescent alcohol use outcomes: initiation and levels of later use/misuse. Based on 131 relatively high-quality studies, 10 of the 12 parenting factors had sound evidence for predicting at least one adolescent alcohol use outcome. Alcohol-specific parenting behaviors (e.g. parental provision of alcohol, rules about alcohol use) yielded larger mean effects on adolescent alcohol use, compared to more general parent-child relational factors (e.g. parental support, parental involvement), with the exception of parental monitoring. Consistent with the family interactional framework for understanding the psychosocial etiological factors for adolescent drug use [38], it is likely that parent-child relational factors would not only have some direct effects on adolescent alcohol misuse, but also serve as important foundational bases upon which other alcohol-specific parenting factors operate as risk or protective factors. For example, rules about adolescent alcohol use are more likely to be protective when established by parents in the context of a good parent-adolescent relationship. Conversely, there is also some evidence suggesting that parental modelling of alcohol misuse may increase their adolescent's risk especially when there is a close parent-child relationship [39].

Below we discuss our findings under the following classes: 1) sound, convergent evidence, whereby the longitudinal associations between the parenting factor and both alcohol use outcomes were significant using both the Stouffer's $p$ and meta-analytic methods of synthesis; 2 ) emerging evidence, whereby the association between the parenting factor and one outcome was significant using both methods of synthesis; and 3) weak, inconsistent evidence, whereby the association between the parenting factor and either one or both outcomes was not significant based on findings from either one or both synthesis methods. Under this classification, 7 parenting factors had a sound, convergent evidence base: (1) provision of alcohol, (2) parental monitoring, (3) favorable attitudes towards alcohol, (4) parental alcohol use, (5) parent-child relationship quality, (6) parental support, and (7) parental involvement. Three factors had an emerging evidence base (rules about alcohol use, 
family conflict, and parental discipline), whereas two others had weak or inconsistent evidence (alcohol-specific communication and general communication).

\section{Factors with a sound, convergent evidence base}

\section{Risk factors that parents should attempt to reduce or avoid}

Of the 12 parenting factors examined in this review, parental provision of alcohol was the risk factor with the largest effect sizes, accounting for $4 \%$ of variance in initiation and $7 \%$ in levels of later use/misuse. Specifically, adolescents whose parents make alcohol accessible or allow them to drink alcohol at home are more likely to start drinking or have alcohol-related problems earlier, and drink more frequently, at higher quantities, and/or have more alcohol-related problems later in life. These findings support the messages for parents proposed by Kaynak and colleagues [17], which include a clear recommendation that parents do not allow their children to drink underage, or provide alcohol for their adolescent at home or for parties. This is a controversial recommendation, especially within cultures that endorse the belief that letting children have a sip of their parents' wine will teach them responsible drinking. However, alcohol socialisation processes differ markedly across cultures (e.g., between northern and southern Europe; $[40,41])$, highlighting the importance of taking cultural differences into account when recommending prevention strategies [41]. As research examining parental provision of alcohol has been primarily conducted in countries with similar attitudes towards alcohol use, more studies are needed across a wider range of countries [42]. Nevertheless, this review provides clear evidence to back up policies and recommendations against parental provision in cultures where tolerance of binge drinking is the norm.

Consistent with Ryan and colleagues' review [14], two other risk factors with a sound evidence base were identified: Favorable attitudes towards alcohol (accounting for more than $1 \%$ and $3 \%$ variance in initiation and later use/misuse respectively), and Parental alcohol use (more than $1 \%$ and $2 \%$ variance respectively). This evidence base substantiates key educational messages to parents about the impact of their alcohol-related attitudes on their child's risk of later alcohol misuse. Moreover, it 
is likely that parents' attitudes interact in complex ways with their own drinking and decisions regarding provision of alcohol to their child, and together, these parental factors may have compounding effects on adolescent alcohol misuse., In order to produce parental behavior change, prevention interventions directed at parents may need to address these factors in combination. Parent or community education campaigns that leverage the well-being of the child (i.e. protect young people from alcohol-related harms) may also serve to motivate behavior change in parents [43].

\section{Protective factors that parents should attempt to increase}

Parental monitoring emerged as the strongest protective factor, accounting for almost $3 \%$ of variance in initiation and $5 \%$ in later use/misuse. Specifically, by being more aware of their adolescents' activities, whereabouts and friends, parents can help protect their adolescents from later alcohol misuse. Monitoring can be challenging to implement successfully in practice, especially during adolescence when young people are seeking increasing autonomy from their parents. Parents often seek guidance in determining the appropriate balance between monitoring that is proactive and stems from trusting their young person, and monitoring that is reactive and reflects overintrusiveness and distrust $[44,45]$. Unfortunately, given the preponderance of measures of monitoring that primarily assess parental knowledge of their child's activities and whereabouts, but not how that knowledge was obtained, the extant literature is not informative for the purpose of translating this evidence base into actionable strategies for parents [46]. The Delphi expert consensus method has been used to redress this limitation [46-48] and delineate strategies that are specific and actionable, bridging the gap between research evidence and practice. Future research is needed to evaluate whether the implementation of such strategies does indeed lead to a reduced risk for later adolescent alcohol misuse.

Three other protective factors with a sound evidence base were identified: Parent-child relationship quality, Parental support, and Parental involvement, each accounting for about $1 \%$ of variance in 
each alcohol use outcome. Although small in their effect sizes, the evidence base supporting the longitudinal association between each of these relational factors and adolescent alcohol misuse is large and robust.

Taken together, the sound evidence base regarding protective parenting factors indicates that parent skills training programs that provide strategies for parents to be aware of and involved in their adolescent's life within the context of a trusting and supportive parent-child relationship are more likely to be effective. While most existing efficacious family-based programs already include a focus on building the parent-child relationship [49-54], they include a substantial intervention component directed at the child, in addition to the parent-intervention component. Hence it remains to be ascertained whether a parent-only intervention that targets the abovementioned evidence-based protective factors will be adequate for reducing adolescent alcohol misuse.

\section{Factors with an emerging evidence base}

Three parenting factors had a sound evidence base supporting their longitudinal link with initiation of adolescent alcohol use, but not with levels of later use or misuse. In particular, in contrast to Ryan and colleagues' review [14] which reported non-significant Stouffer's ps for the associations between rules about alcohol use and both outcomes, the current meta-analysis indicates that having clear rules against adolescent alcohol use can have a protective effect against underage drinking (accounting for almost $4 \%$ variance in initiation). Likewise, although the earlier review [14] failed to find significant effects of family conflict on either adolescent alcohol use outcome, the current metaanalysis indicates that family conflict may increase risk for early alcohol initiation (1\% variance). Consistent with the earlier review [14], the evidence base for parental discipline continues to suggest that establishing clear and consistent rules for adolescents' behaviour generally may have a protective effect against early initiation, although the amount of variance accounted for is less than half a percent. 
For all three parenting factors, the evidence base remains equivocal for predicting levels of later alcohol use/misuse. Although the Stouffer's ps were significant in all cases, a meta-analysis of a small subset revealed a non-significant mean effect (family conflict), or could not be conducted due to inadequate eligible associations (rules about alcohol use and parental discipline). Clearly more longitudinal studies are required to clarify whether the longitudinal associations between these factors and adolescent alcohol misuse are specific to early initiation, and to disentangle the possible reasons behind this differentiation of effects.

\section{Factors with a weak evidence base}

Finally, there was insufficient evidence to suggest that alcohol-specific communication and general communication are longitudinally associated with adolescent alcohol misuse. Findings regarding alcohol-specific communication are particularly notable in highlighting that not all alcohol-specific communication between parents and adolescents is protective. Indeed, parental disclosure of negative experiences with alcohol (in themselves or others) may in fact increase risk for adolescent alcohol misuse [32]. Handley and Chassin [32] proposed that such parent-child communication about alcohol may inadvertently enhance adolescents' positive views of drinking by normalizing the experience, especially if the parent jokes or laughs about the experience.

The equivocal findings regarding general parent-child communication were unexpected, given the significant findings in Ryan and colleagues' review[14], and the importance of communication in the parent-child relationship. It is possible that measures of parent-child verbal communication in general [34] or general discussions about daily issues [35] do not tap adequately into the construct of parent-child communication that is expected to be protective. In contrast, measures that assess adolescent disclosure to parents [33] and positive aspects of communication (e.g. Parent-Adolescent Communication Scale $[55,56])$ may better reflect the type and quality of parent-child communication that operates in the context of a close parent-child relationship to protect against alcohol misuse by the adolescent. Future research should take into account such nuances and select 
measures of communication that more accurately assess the positive attachment-related quality of parent-child communication.

\section{Moderation effects}

Our review also identified some noteworthy moderators. Firstly, while included studies had a minimum interval of one year between the measurement of parenting and the adolescent alcohol misuse outcome, the longest follow-up interval was 26 years [36], with parent-child relationship quality found to significantly predict alcohol-related problems in adulthood. Notwithstanding, follow-up interval was found to significantly moderate the association between 5 parenting factors and adolescent alcohol misuse, with smaller effect sizes in studies with longer intervals: favorable attitudes towards alcohol and family conflict on initiation; parental support and parental involvement on later use/misuse; and parent-child relationship on both outcomes. These findings indicate that while the developmental salience of parenting may fade over time, parenting factors can influence young people's alcohol use outcomes over the longer term. Secondly, similar moderation effects were found for child age at baseline, whereby studies with younger children at baseline yielded smaller effect sizes, for 3 parenting factors: family conflict and parent-child relationship quality on initiation; and parental involvement on later use/misuse. Studies with longer follow-up intervals are also likely to have recruited younger children at baseline, hence the latter findings may be an artefact of the former rather than a reflection of developmental differences. To address this question, more longitudinal studies are required to ensure that meta-regression analyses are powered to examine these two moderators in one model.

The different rates of alcohol misuse and related harms in male and female adolescents $[57,58]$ underscore the need to better understand the etiological factors underlying the development of these problems in boys versus girls. The current review conducted moderator analyses for 7 of the 12 parenting factors (for at least one or both outcomes): Family conflict, General communication, Parent-child relationship quality, Parental alcohol use, Parental involvement, Parental monitoring, 
and Provision of alcohol. Of these, significant moderation by child gender was found only for parentchild relationship quality and parental monitoring, suggesting that girls may be more sensitive to the effects of both factors, in terms of their risk for early initiation. These findings support the rationale for Schinke and colleagues' gender-specific intervention focusing on fostering mother-daughter closeness and parental monitoring, which has been found to be effective in reducing levels of alcohol use at 1- and 2-year follow-up [52-54]. More studies examining the longitudinal associations specific for each gender group, and even the different parent-child gender combinations, are required to further examine the potential moderator effects of parent and child gender.

Finally, although we conducted analyses to examine moderation by type of outcome measure for the associations between all but two parenting factors (Alcohol-specific communication and Rules about alcohol) and at least one outcome, significant moderation was found only in the associations between favorable attitudes towards alcohol and initiation, and between parent-child relationship quality and both initiation and levels of later use/misuse. The number of non-significant moderation findings suggest that most of the examined longitudinal associations between parenting factors and adolescent alcohol misuse outcomes may not be affected by the type of outcome measures used. Nonetheless, significant moderation analyses indicate that, unsurprisingly, both favorable parental attitudes towards alcohol and parent-child relationship quality have a stronger association with alcohol initiation when the latter is assessed using measures of alcohol-related outcomes other than onset, such as frequency/quantity of use and excessive/binge drinking. This finding may be partly due to methodological factors (e.g. onset is measured as a dichotomous variable whereas alcoholrelated outcomes are usually measured as continuous variables, hence affording more variance). These findings may also indicate that by establishing a close relationship with their child and clearly conveying disapproval of adolescent alcohol use, parents can protect their child from alcohol misuse in early adolescence by delaying onset to an extent, but perhaps more significantly, they can reduce the harmful outcomes associated with underage drinking (even if they cannot totally prevent alcohol 
initiation). This concurs with recommendations from both the Australian [8] and UK [8] guidelines that if young people aged between 15-17 years do drink, it should be done under adult supervision, in a safe environment and only at a low-risk level. The other significant moderation effect found suggests that a good parent-child relationship is protective against levels of later use/misuse after mid-adolescence, but this protective effect may be more evident on frequency or quantity of alcohol used, compared to other potentially more severe indicators of harms (e.g. frequency of drunkenness, excessive/binge drinking, abuse/dependence).

\section{Parent gender-specific associations}

We conducted supplementary meta-analyses to examine the associations between 7 of the 12 factors and at least one outcome, for mothers and fathers separately: Alcohol-specific communication (initiation only), Favorable attitudes toward alcohol use (both outcomes), General communication (use/misuse only), Parent-child relationship quality (both outcomes), Parental alcohol use (both outcomes), Parental discipline (initiation only), and Parental support (both outcomes). Of the 11 sets of analyses conducted, differences were observed for fathers versus mothers for four sets, all of which were in predicting initiation: parental alcohol use; favorable attitudes towards alcohol use; parental support and parental discipline. Albeit preliminary, these findings suggest that mothers may have a more significant impact on their child's early initiation through the relational, general parenting factors (i.e. being supportive and having clear and consistent disciplinary boundaries for their child's behavior); whereas fathers may have a more significant impact through the alcohol-specific factors (parental drinking and favorable attitudes towards alcohol). Future research is needed to test these hypotheses, and further examine the associations between parenting factors and adolescent alcohol misuse in the context of different family compositions (e.g. same-sex parents, separated parents). Further research should also examine whether differences in associations may reflect differences in quality of reporting by fathers versus mothers. 


\section{Strengths and limitations}

One of the most significant strengths of this review is its inclusion of meta-analyses of a subset of studies that provided suitable data, to supplement the use of Stouffer's $p$ to synthesize a wider range of eligible studies. Importantly, this is the first meta-analysis of observational studies examining longitudinal associations between parenting factors and adolescent alcohol misuse. This review also included a more comprehensive coverage of any parenting factors that are potentially modifiable, and a clear systematic review protocol, including the use of the rigorous PRISMA methodology, and a more comprehensive search strategy, which identified a greater number of relevant studies than earlier reviews. Moreover, although early initiation is clearly correlated with levels of use/misuse, examining them as separate outcomes in this review revealed interesting unique associations that each has with the various parenting factors.

Several limitations to this review should be noted. Firstly, our review is subject to the potential limitations of any meta-analysis of observational studies, e.g. inherent biases and differences in the design of included studies may render the computation of a single summary estimate potentially misleading [59]. In trying to reduce this risk, we pre-specified clear criteria for including studies in meta-analysis, including the requirement that data be reported without adjustment for covariatesthis was necessary because most studies adjusted for different combinations of covariates, precluding a meaningful statistical synthesis. Unfortunately, this resulted in the exclusion of $60 \%$ of associations from meta-analysis, which meant that some meta-analyses included a very small number of independent studies (minimum=2). Consequently, some meta-analyses may have been underpowered to detect significant associations (e.g. between General communication and alcohol initiation). Moreover, including longitudinal associations from primary studies that have not been adjusted for covariates also precludes us from drawing inferences of causality or directionality, and the magnitudes of mean effect sizes may have been inflated. Nonetheless, given the recommendation that there be a minimum of three waves of assessments of both predictor and outcome variables to determine directionality of associations [60], the best way to conduct meta- 
analyses of such complex datasets remains to be clarified. In the meantime, the current review echoes the recommendation from other recent reviews that future longitudinal studies follow some standardized reporting requirements, to facilitate meta-analytic syntheses [22, 23]. For example, follow-up outcomes could be reported with adjustment for baseline levels of alcohol use only, in addition to other models that are adjusted for other covariates of interest to that study. Alternatively, authors of independent studies may consider a collaborative pooling of data, as has been done in other research fields [61].

Another limitation of our findings is that they relate primarily to developed countries where the studies were conducted - i.e. US, Europe/UK, Australia and New Zealand, and may not be generalizable to developing countries or other cultures. Although various primary studies examined the effects of parenting on adolescent alcohol misuse separately for different ethnic groups (e.g. [62-64]) or with a specific focus on ethnic minorities (e.g. $[65,66])$, more research is required across a broad range of developed and developing countries with differing cultural attitudes to alcohol to examine the similarities and differences in the role of parenting in adolescent alcohol use.

The substantial heterogeneity across studies in most of the meta-analyses conducted (20 of the 21 meta-analyses had more than $50 \%$ heterogeneity) is another noteworthy limitation. Although it is not ideal to conduct meta-analyses on heterogeneous studies, in order to advance the field, researchers have to start moving from purely qualitative/narrative syntheses to quantitative syntheses. We tried to address the heterogeneity by relying on random-effects models, and conducting post-hoc sensitivity analyses which excluded potentially outlying studies (e.g. which relied on substantially different measures [35], or had an extraordinarily long follow-up interval [36]), and effectively reduced the heterogeneity in some cases. Moderator analyses were also conducted to generate hypotheses about potential sources of heterogeneity that future research can explore. Most studies did not use standardized, validated measures to assess parenting behaviors, resulting in marked discrepancies in the content of measures categorized as measuring a purportedly similar 
parenting construct. This is likely to have contributed to the high heterogeneity observed. We attempted to manage this by providing clear and narrower definitions of parenting factors; e.g. having five specific factors to assess the parent-child relationship-Parent-child relationship quality, Parental support, Parental involvement, Parental discipline, and General communication-as opposed to the global 'parent-child relationship construct' as used in Visser and colleagues' review [19], which may have obscured significant associations for some (e.g. Parental support) but not other (e.g. General communication) specific parent-child relational factors. This also highlights the need for future studies to rely on psychometrically sound, clearly operationalized and validated parenting measures.

Furthermore, given our aim to provide a macro-level synthesis of the burgeoning, diverse evidence base, it was beyond the scope of this review to examine potential mediational effects. Given the multiple analyses conducted, post-hoc and supplementary analyses should be considered as hypothesis-generating analyses, to guide future research. It should also be noted that the statistically non-significant associations found in this review could be due to data insensitivity or lack of statistical power; further research examining those associations are required. Finally, the causal role of the parenting factors examined in this review remains to be determined by well-designed randomized controlled trials that directly assess their mediating effects [67].

\section{Implications for future prevention practice and policy}

In considering the implications of our findings for practice and policy, the small effect sizes that emerged from our meta-analyses (accounting for only $1 \%$ to $7 \%$ variance) beg the more fundamental question: are parents important for the prevention of adolescent alcohol misuse? The magnitude of these effects is similar to those reported in meta-analyses of parenting factors on child internalizing $[22,23,68-70]$ and externalizing [71] problems. While such findings seem to suggest that parenting factors are not that important for a range of child outcomes, it is important to consider a few caveats to such a conclusion. Firstly, the effects reported involved specific, narrowly- 
defined parenting factors, so it is possible that different factors may have additive or multiplicative effects on adolescent alcohol use (e.g. parental drinking and parent-child relationship quality [71]). Secondly, parenting factors may also have additional indirect effects on adolescent alcohol use through other risk or protective factors that have a significant direct effect; e.g. parental support was found to prospectively influence the development of peer relationships which in turn predicted subsequent levels of problem-drinking behavior [72]. Thirdly, Stockings and colleagues' systematic review of reviews revealed that amongst existing psychologically-based prevention interventions to reduce alcohol use in young people, family-based programs (most of which include separate and combined parent and child components) were the only type of interventions with small but sustained effects; in contrast to interventions targeting populations of young people primarily (e.g. school-based interventions), which have inconclusive or mixed evidence [10]. One possible explanation for this pattern of findings is that having a parent component which focuses on building parenting skills and parent-child relationships is a critical element of the family-based interventions, relative to interventions that target only the child. If this is true, then evidence from intervention research would argue against a conclusion that parents do not matter in prevention of adolescent alcohol misuse. Nonetheless, further research on new and existing interventions that focus primarily on building parents' skills (across the modifiable parenting factors examined in this review), without direct intervention with young people, is required to more clearly demonstrate the preventive importance of parenting factors.

Taken together, it would seem amiss to devalue the importance of parents and parenting in prevention of adolescent alcohol misuse, especially given the prominent role parents play in a young person's life even throughout adolescence, and parents' intrinsic motivation to facilitate their child's well-being and prevent harms from alcohol misuse. At the nexus of policy and practice, parents are the primary implementers of national guidelines and legislation regarding alcohol use by young people, so it is important that they are adequately equipped to fulfil this important role successfully. Hence interventions designed to upskill parents in these evidence-based parenting factors are 
urgently needed. Given the rapid advances in technology, tailored online programs may be a costeffective universal approach to equip parents in their role in the prevention of adolescent alcohol misuse [73].

\section{Conclusions}

This systematic review and collection of meta-analyses revealed a set of modifiable parenting factors that have a longitudinal association with adolescent alcohol initiation and levels of use/misuse. While there is a need for more well-designed longitudinal studies using clearly-defined and validated measures, and which report their data in ways that facilitate meta-analyses, findings from this review support the translation of the sound evidence base on the various parenting factors examined here into prevention interventions to upskill parents. 


\section{Acknowledgements}

Author Yap received salary support from a National Health and Medical Research Council (NHMRC) Career Development Fellowship (APP1061744). Author Jorm received salary support from an NHMRC Senior Principal Research Fellowship (APP1059785). The funding sources did not have any role in the conduct or publication of this study. 


\section{References}

1. Degenhardt L, Stockings E, Patton G, Hall WD, Lynskey M. The increasing global health priority of substance use in young people. The Lancet Psychiatry. 2016 3//;3(3):251-64.

2. WHO. Global strategy to reduce the harmful use of alcohol. Geneva: World Health Organization, 2010.

3. Hall WD, Patton G, Stockings E, Weier M, Lynskey M, Morley KI, et al. Why young people's substance use matters for global health. The Lancet Psychiatry. 2016 3//;3(3):265-79.

4. Lubman DI, Bonomo Y, Yücel M. Drug use in young people: Short-term effects and long-term harms. In: Gilvarry E, McArdle P, editors. Clinics in Developmental Medicine Alcohol, drugs and young people: Clinical Approaches. London: MacKeith Press; 2007. p. 18-50.

5. Lubman DI, Hides L, Yücel M, Toumbourou J. Intervening early to reduce developmentally harmful substance use amongst youth populations. Medical Journal of Australia. 2007;187:S22-S5.

6. Bonomo YA, Coffey C, Wolfe R, Lynskey M, Bowes G, Patton G. Adverse outcomes of alcohol use in adolescents. Addiction. 2001;96:1485-96.

7. U.S. Department of Health and Human Services. The Surgeon General's call to action to prevent and reduce underage drinking: A Guide to Action for Communities2007. Available from: https://www.surgeongeneral.gov/library/calls/underage-drinking-community-guide.pdf (Archived by WebCite ${ }^{\circledR}$ at http://www.webcitation.org/6mkU7LktC).

8. Donaldson L. Guidance on the Consumption of Alcohol by Children and Young People. A report by the Chief Medical Officer2009. Available from: http://www.cph.org.uk/wpcontent/uploads/2013/09/Guidance-on-the-consumption-of-alcohol-by-children-and-youngpeople.pdf (Archived by WebCite ${ }^{\circledR}$ at http://www.webcitation.org/6mkUEVtF1).

9. National Health \& Medical Research Council. Australian Guidelines to Reduce Health Risks from Drinking Alcohol. Canberra 2009.

10. Stockings E, Hall WD, Lynskey M, Morley KI, Reavley N, Strang J, et al. Prevention, early intervention, harm reduction, and treatment of substance use in young people. The Lancet Psychiatry. 2016 3//;3(3):280-96.

11. Foxcroft DR, Tsertsvadze A. Universal family-based prevention programs for alcohol misuse in young people. Cochrane Database of Systematic Reviews. 2011;9. PubMed PMID: CD009308. 12. Stevens MM, Olson AL, Gaffney CA, Tosteson TD, Mott LA, Starr P. A pediatric, practicebased, randomized trial of drinking and smoking prevention and bicycle helmet, gun, and seatbelt safety promotion. Pediatrics. 2002 Mar;109(3):490-7. PubMed PMID: 11875146. Epub 2002/03/05. eng.

13. Spoth R, Lopez Reyes M, Redmond C, Shin C. Assessing a public health approach to delay onset and progression of adolescent substance use: latent transition and log-linear analyses of longitudinal family preventive intervention outcomes. Journal of consulting and clinical psychology. 1999 Oct;67(5):619-30. PubMed PMID: 10535229. Epub 1999/10/27. eng.

14. Ryan SM, Jorm AF, Lubman DI. Parenting factors associated with reduced adolescent alcohol use: a systematic review of longitudinal studies. The Australian and New Zealand journal of psychiatry. 2010;44(9):774-83.

15. Hibell B, Guttormsson U, Ahlstrom T, Balakireva O, Bjarnason T, Kokkevi K, et al. The 2007 ESPAD Report: Substance use amongst students in 35 European countries. Stockholm: The Swedish Council for Information on Alcohol and Other Drugs, 2009.

16. Gilligan C, Kypri K, Lubman D. Changing parental behaviour to reduce risky drinking among adolescents: current evidence and future directions. Alcohol and alcoholism (Oxford, Oxfordshire). 2012 May-Jun;47(3):349-54. PubMed PMID: 22395229. Epub 2012/03/08. eng.

17. Kaynak O, Winters KC, Cacciola J, Kirby KC, Arria AM. Providing alcohol for underage youth: what messages should we be sending parents? J Stud Alcohol Drugs. 2014 Jul;75(4):590-605. PubMed PMID: 24988258. Pubmed Central PMCID: PMC4108600. Epub 2014/07/06. eng. 18. Rossow I, Keating P, Felix L, McCambridge J. Does parental drinking influence children's drinking? A systematic review of prospective cohort studies. Addiction. 2016;111(2):204-17. 
19. Visser L, de Winter AF, Reijneveld SA. The parent-child relationship and adolescent alcohol use: a systematic review of longitudinal studies. BMC Public Health. 2012;12:886. PubMed PMID: 23083405. Pubmed Central PMCID: 3534438. Epub 2012/10/23. eng.

20. Čablova L, Pazderkova K, Miovsky M. Parenting styles and alcohol use among children and adolescents: A systematic review. Drugs: Education, Prevention \& Policy. 2014;21(1):1-13. PubMed PMID: 93891869.

21. Hayden JA, Côté P, Bombardier C. Evaluation of the quality of prognosis studies in systematic reviews. Annals of internal medicine. 2006;144(6):427-37.

22. Yap MB, Pilkington PD, Ryan SM, Jorm AF. Parental factors associated with depression and anxiety in young people: a systematic review and meta-analysis. Journal of Affective Disorders. 2014 Mar;156:8-23. PubMed PMID: 24308895. English.

23. Yap MBH, Jorm AF. Parental factors associated with childhood anxiety, depression, and internalizing problems: A systematic review and meta-analysis. J Affect Disord. 2015 Apr 1;175C:42440. PubMed PMID: 25679197. Epub 2015/02/14. Eng.

24. Cairns KE, Yap MB, Pilkington PD, Jorm AF. Risk and protective factors for depression that adolescents can modify: a systematic review and meta-analysis of longitudinal studies. J Affect Disord. 2014 Dec;169:61-75. PubMed PMID: 25154536. Epub 2014/08/27. eng.

25. Stouffer SA, Suchman EA, DeVinney LC, Star SA, Williams RMJ. The American soldier: Vol. 1: Adjustment during Army Life.: Princeton, Princeton University Press; 1949.

26. Comprehensive Meta Analysis. (Version 3) [computer software]. 3 ed. Englewood, NJ: Biostat; 2013.

27. Cohen J. A power primer. Psychological Bulletin. 1992;112:155-9.

28. Higgins JP, Thompson SG, Deeks JJ, Altman DG. Measuring inconsistency in meta-analyses. BMJ. 2003;327:557-60.

29. Egger M, Smith G, Phillips A. Meta-analysis: principles and procedures. BMJ 1997;315:1533-7.

30. Duval SJ, Tweedie RL. A non-parametric trim and fill method of accounting for publication bias in meta-analysis. J Am Stat Assoc. 2000;95:89-98.

31. Donovan JE, Molina BSG. Childhood risk factors for early-onset drinking. Journal of studies on alcohol and drugs. 2011;72(5):741-51.

32. Handley ED, Chassin L. Alcohol-specific parenting as a mechanism of parental drinking and alcohol use disorder risk on adolescent alcohol use onset. Journal of studies on alcohol and drugs. 2013;74(5):684-93.

33. Abar CC, Jackson KM, Colby SM, Barnett NP. Parent-child discrepancies in reports of parental monitoring and their relationship to adolescent alcohol-related behaviors. Journal of youth and adolescence. 2015;9:1688-701.

34. Jordan LC, Lewis ML. Paternal Relationship Quality as a Protective Factor: Preventing Alcohol Use Among African American Adolescents. Journal of Black Psychology. 2005;31(2):152-71. PubMed PMID: 2005-03099-003.

35. Cookston JT, Finlay AK. Father involvement and adolescent adjustment: Longitudinal findings from Add Health. Fathering. 2006;4:137-58.

36. Maggs JL, Patrick ME, Feinstein L. Childhood and adolescent predictors of alcohol use and problems in adolescence and adulthood in the National Child Development Study. Addiction (Abingdon, England). 2008 May;103 Suppl 1:7-22. PubMed PMID: 18426537. Epub 2008/05/28. eng. 37. Latendresse SJ, Rose RJ, Viken RJ, Pulkkinen L, Kaprio J, Dick DM. Examining the etiology of associations between perceived parenting and adolescents' alcohol use: Common genetic and/or environmental liabilities. Journal of studies on alcohol and drugs. 2010;71(3):313-25.

38. Brook JS, Brook DW. The psychosocial etiology of adolescent drug use: A family interactional approach. Genetic, Social \& General Psychology Monographs. 1990 05;116(2):111.

39. Andrews JA, Hops H, Duncan SC. Adolescent modeling of parent substance use: The moderating effect of the relationship with the parent. Journal of Family Psychology. 1997;11(3):25970. PubMed PMID: 1997-05658-001. 
40. Rolando S, Beccaria F, Tigerstedt C, Törrönen J. First drink: What does it mean? The alcohol socialization process in different drinking cultures. Drugs: Education, Prevention and Policy. 2012 2012/06/01;19(3):201-12.

41. Velleman R. How do Children and Young People Learn About Alcohol: A Major Review of the Literature for the Joseph Rowntree Foundation. Bath, U.K.: University of Bath, 2009.

42. Jones SC. Parental provision of alcohol: a TPB-framed review of the literature. Health promotion international. 2015 Apr 23. PubMed PMID: 25908595. Epub 2015/04/25. Eng.

43. Hawkins JD, Catalano RF, Miller JY. Risk and protective factors for alcohol and other drug problems in adolescence and early adulthood: implications for substance abuse prevention. Psychol Bull. 1992;112:64 - 105. PubMed PMID: doi:10.1037/0033-2909.112.1.64.

44. Stattin H, Kerr M. Parental Monitoring: A Reinterpretation. Child Development.

2000;71:1072 - 85. PubMed PMID: doi:10.1111/1467-8624.00210.

45. Abar C, Jackson $\mathrm{K}$, Wood M. Examining proactive versus reactive parental monitoring in the context of alcohol use across adolescence. Alcoholism: Clinical and Experimental Research. 2011;35:29A.

46. Yap MB, Pilkington PD, Ryan SM, Kelly CM, Jorm AF. Parenting strategies for reducing the risk of adolescent depression and anxiety disorders: a Delphi consensus study. Journal of Affective Disorders. 2014 Mar;156:67-75. PubMed PMID: 24359862. English.

47. Jorm AF. Using the Delphi expert consensus method in mental health research. Australian and New Zealand Journal of Psychiatry. 2015 October 1, 2015;49(10):887-97.

48. Ryan SM, Jorm AF, Kelly C, Hart L, Morgan AJ, Lubman DI. Parenting to reduce adolescent alcohol consumption: A Delphi consensus study. BMC Public Health. 2011;11(1):13.

49. Brody GH, Murry VM, McNair L, Chen Y-F, Gibbons FX, Gerrard M, et al. Linking Changes in Parenting to Parent-Child Relationship Quality and Youth Self-Control: The Strong African American Families Program. Journal of Research on Adolescence. 2005;15(1):47-69. PubMed PMID: 200502820-003.

50. Spoth RL, Redmond C, Shin C. Randomized trial of brief family interventions for general populations: Adolescent substance use outcomes 4 years following baseline. Journal of Consulting \& Clinical Psychology. 2001;69:627-42.

51. Bauman KE, Ennett ST, Foshee VA, Pemberton M, King TS, Koch GG. Influence of a Family Program on Adolescent Smoking and Drinking Prevalence. Prev Sci. 2002;3(1):35-42.

52. Schinke SP, Cole KC, Fang L. Gender-specific intervention to reduce underage drinking among early adolescent girls: A test of a computer-mediated, mother-daughter program. Journal of Studies on Alcohol and Drugs. 2009;70:70-7.

53. Schinke SP, Fang L, Cole KC. Computer-delivered, parent-involvement intervention to prevent substance use among adolescent girls. Preventive Medicine. 2009 Nov;49(5):429-35. PubMed PMID: ISI:000271849200016. English.

54. Schinke SP, Fang L, Cole KC. Preventing substance use among adolescent girls: 1-year outcomes of a computerized, mother-daughter program. Addictive Behaviors. 2009;34(12):1060-4. 55. Nash SG, McQueen A, Bray JH. Pathways to adolescent alcohol use: family environment, peer influence, and parental expectations. The Journal of adolescent health : official publication of the Society for Adolescent Medicine. 2005 Jul;37(1):19-28. PubMed PMID: 15963903. Epub 2005/06/21. eng.

56. Ohannessian CM. Parental problem drinking and adolescent psychosocial adjustment: The mediating role of adolescent-parent communication. Journal of Research on Adolescence. 2012;22(3):498-511. PubMed PMID: 2012-22489-010.

57. Australian Institute of Health and Welfare. National Drug Strategy Household Survey detailed report: 2013. Canberra AlHW, 2014.

58. 2013 National Survey on Drug Use and Health Public Use File [Internet]. Substance Abuse and Mental Health Services Administration. 2013. 
59. Stroup DF, Berlin JA, Morton SC, et al. Meta-analysis of observational studies in epidemiology: A proposal for reporting. Jama. 2000;283(15):2008-12.

60. Curran PJ, Bollen KA. The best of both worlds: Combining autoregressive and latent curve models. In: Collins LM, Sayer AG, editors. New methods for the analysis of change. Washington, DC: American Psychological Association.; 2001. p. 105-36.

61. Anstey KJ, Bielak AAM, Birrell CL, Browning CJ, Burns RA, Byles J, et al. Understanding ageing in older Australians: The contribution of the Dynamic Analyses to Optimise Ageing (DYNOPTA) project to the evidence base and policy. Australasian Journal on Ageing. 2011;30:24-31.

62. Barnes GM, Farrell MP, Banerjee S. Family influences on alcohol abuse and other problem behaviors among Black and White adolescents in a general population sample. Journal of Research on Adolescence. 1994;4(2):183-201. PubMed PMID: 1994-41494-001.

63. Kopak AM, Chen AC, Haas SA, Gillmore MR. The importance of family factors to protect against substance use related problems among Mexican heritage and White youth. Drug and alcohol dependence. 2012 Jul 1;124(1-2):34-41. PubMed PMID: 22222253. Pubmed Central PMCID: Pmc3337392. Epub 2012/01/10. eng.

64. Peterson PL, Hawkins JD, Abbott RD, Catalano RF. Disentangling the effects of parental drinking, family management, and parental alcohol norms on current drinking by black and white adolescents. Journal of Research on Adolescence. 1994;4(2):203-27.

65. Cleveland MJ, Gibbons FX, Gerrard M, Pomery EA, Brody GH. The impact of parenting on risk cognitions and risk behavior: a study of mediation and moderation in a panel of African American adolescents. Child development. 2005 Jul-Aug;76(4):900-16. PubMed PMID: 16026504. Epub 2005/07/20. eng.

66. Shin Y, Lee JK, Lu Y, Hecht ML. Exploring parental influence on the progression of alcohol use in Mexican-heritage youth: A latent transition analysis. Prevention Science. 2016;17:188-98.

67. Sandler IN, Schoenfelder EN, Wolchik SA, MacKinnon DP. Long-term impact of prevention programs to promote effective parenting: Lasting effects but uncertain processes. Annual review of psychology. 2011;62(1):299-329.

68. Amato PR. Children of divorce in the 1990s: An update of the Amato and Keith (1991) metaanalysis. J Fam Psychol. 2001;15:355-70.

69. McLeod BD, Weisz JR, Wood JJ. Examining the association between parenting and childhood depression: A meta-analysis. Clin Psychol Rev. 2007;27(8):986-1003.

70. McLeod BD, Wood JJ, Weisz JR. Examining the association between parenting and childhood anxiety: A meta-analysis. Clin Psychol Rev. 2007;27(2):155-72.

71. Rothbaum F, Weisz JR. Parental caregiving and child externalizing behavior in nonclinical samples: A meta-analysis. Psychological Bulletin. 1994;116:55-74.

72. Coker JK, Borders LD. An analysis of environmental and social factors affecting adolescent problem drinking. Journal of Counseling \& Development. 2001;79:200-8.

73. Yap MBH, Jorm AF, Bazley R, Kelly CM, Ryan SM, Lubman D. Web-based parenting program to prevent adolescent alcohol misuse: rationale and development. Australasian Psychiatry.

2011;19(4):339-44. Epub 24 August 2011. 

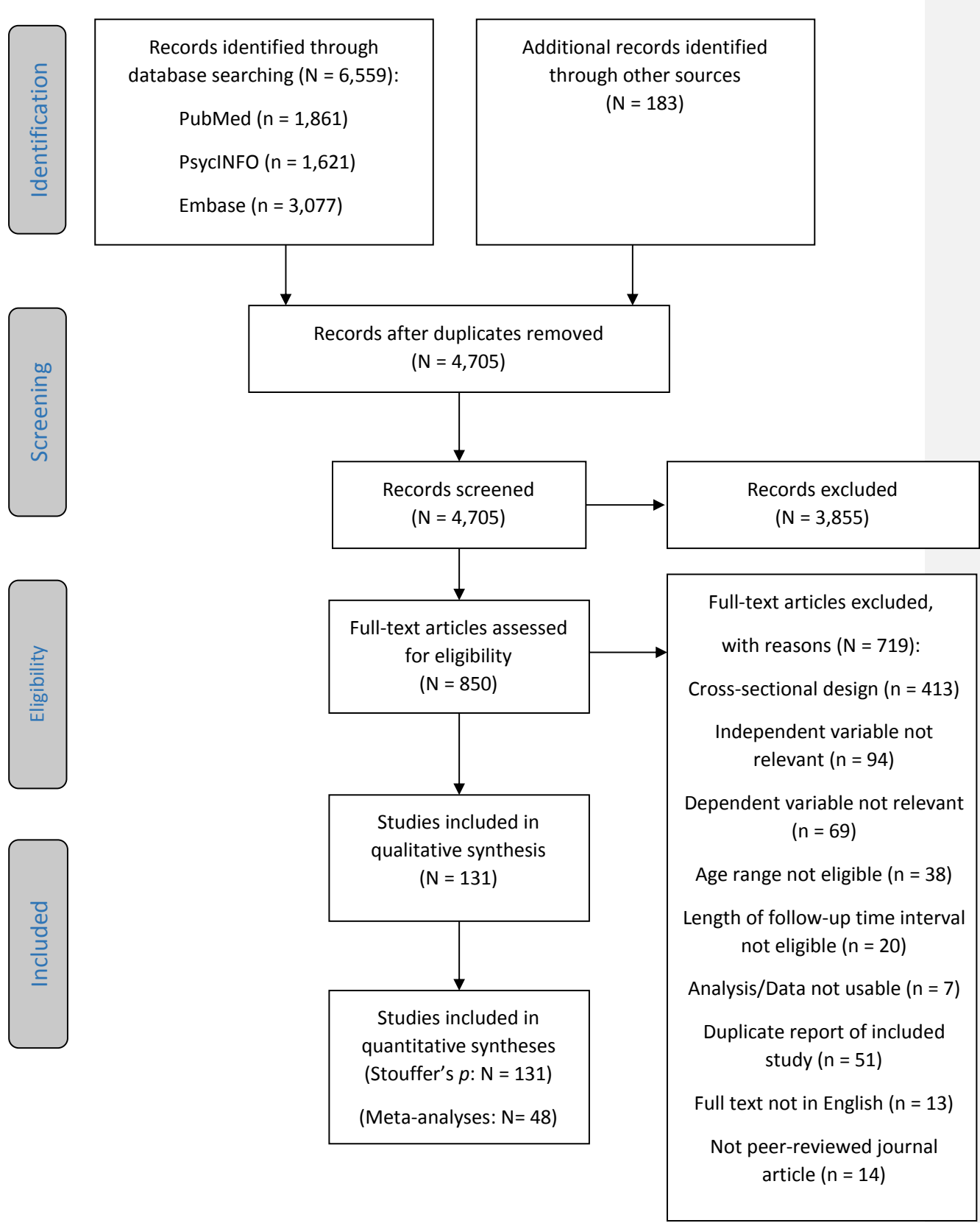

Figure 1. Systematic literature search process. 


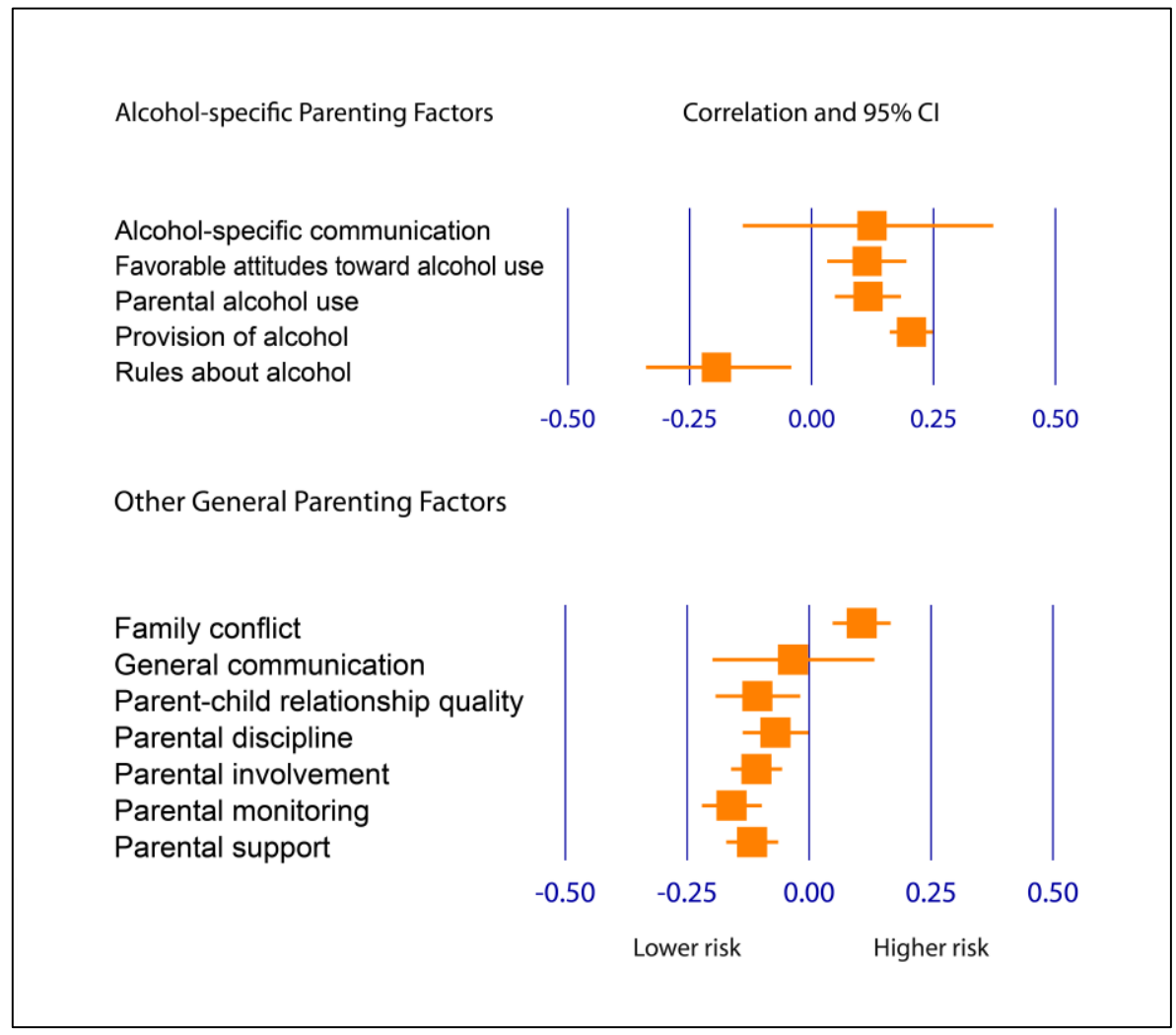

Figure $2 a$. Mean effect sizes for each association between a specific parenting factor and initiation of alcohol use.

Note: Effect sizes were not pooled across different parenting factors. 


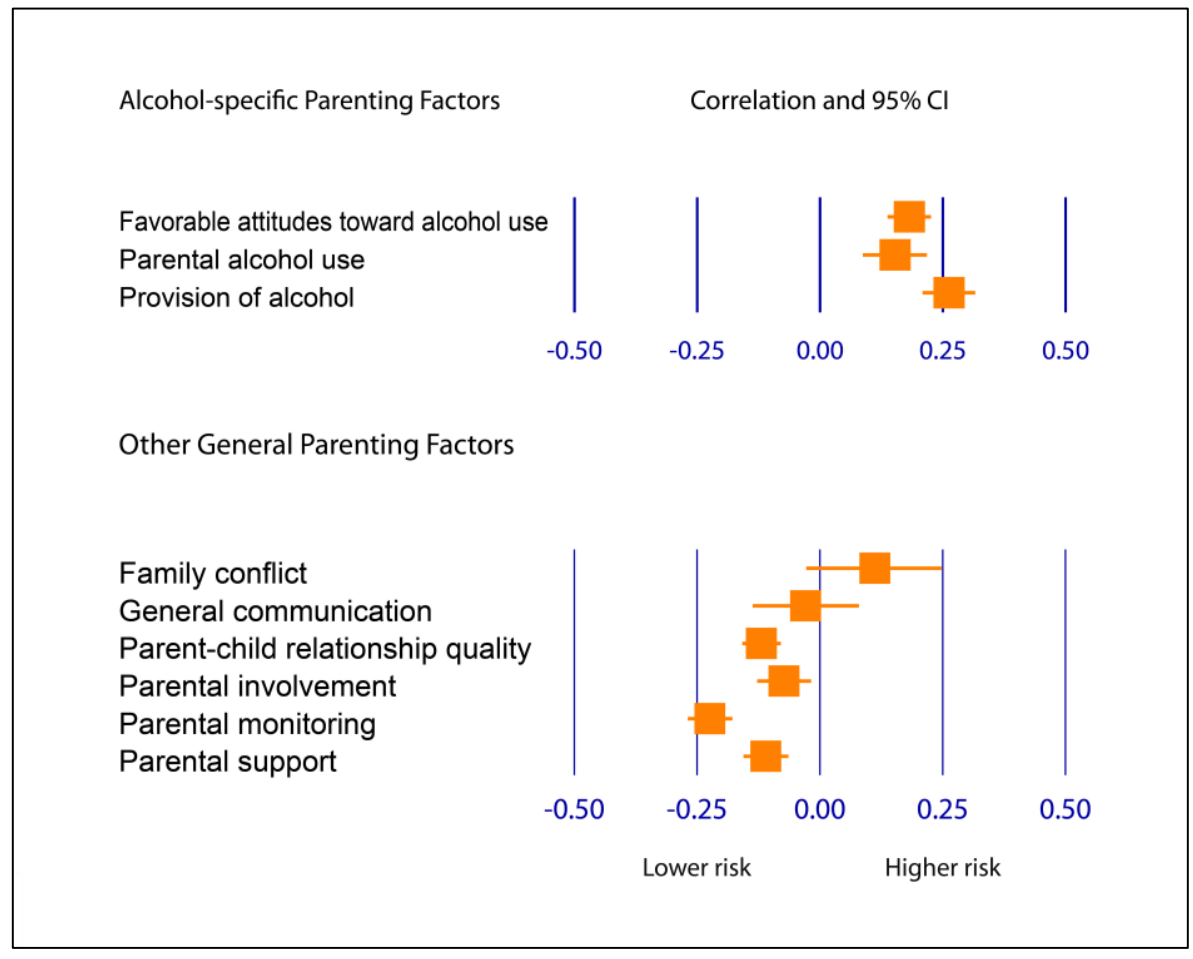

Figure $2 b$. Mean effect sizes for each association between a specific parenting factor and levels of later alcohol use/misuse.

Notes: Effect sizes were not pooled across different parenting factors. Three factors (alcohol-specific communication, parental discipline, and rules about alcohol use) are not shown in this plot as they had insufficient studies for conducting meta-analyses. 
Table 1. Definitions of parenting factors and hypothesized associations.

\begin{tabular}{|c|c|c|}
\hline Parenting factor & Definition & $\begin{array}{l}\text { Hypothesized associations } \\
\text { with initiation of alcohol } \\
\text { use and levels of later } \\
\text { alcohol use/misuse }\end{array}$ \\
\hline
\end{tabular}

\section{Alcohol-specific parenting factors}

Alcohol-specific communication

Favorable attitudes toward alcohol use

Parental alcohol use ${ }^{\text {a }}$

Provision of alcoho

Rules about alcohol use

The extent to which parents set explicit rules to prevent unsupervised drinking and/or limit the drinking amount of their adolescent child

The frequency and/or quality of parent-child discussions about alcohol, including negative consequences of alcohol misuse, parents' personal experiences of alcohol use, and suggestions about responsible drinking

The degree to which parents hold a favorable attitude toward or approve of adolescent alcohol use, including underage drinking in general and the drinking behaviors of their own adolescent child

The frequency and/or intensity of parents' drinking behaviors which can potentially be observed and learned by their adolescent child

The degree to which parents make alcohol accessible or allow their adolescent child to drink alcohol at home

Negative

Positive

Negative

Positive

Positive

Positive ension within the family environr

Negative

The frequency, quality, and/or satisfaction of communication between parents and their adolescent child, with conversations ranging from purely factual topics to highly emotional sharing

Parent-child relationship quality

Parental discipline ${ }^{\mathrm{b}}$
The closeness, mutual attachment, and positive interactions perceived by parents and/or their adolescent child

The degree to which parents are capable of regulating their child's behaviors in an adequate and/or consistent manner, such as reasonably restricting their child's activities, and establishing strict but consistent rules
Negative

Negative 
Parental involvement

The frequency that parents and their adolescent child engage in shared activities, such as playing games, watching

television, shopping, and having dinner together

Parental monitoring

The extent to which parents know about their child's activities, whereabouts, and friends
Negative

Negative

Negative

Parental support

The extent to which parents provide emotional and/or instrumental support to their adolescent child, such as warmth, love, acceptance, encouragement, praise, practical help and guidance

Note: Favorable attitudes toward alcohol use, Parental alcohol use, and Parental discipline were named Disapproval of adolescent drinking (reversely coded), Parental modeling, and General discipline respectively in Ryan et al. (2010). These factors were renamed to better reflect the studies categorised within each parenting factor in the current review. Most of the definitions were also slightly modified to include specific examples to clarify the measures captured within each parenting factor.

${ }^{a}$ Parental alcohol use does not include variables that represent problematic drinking, such as binge drinking, excessive drinking, abuse/dependency, and alcohol-related problems. This factor only refers to frequency and/or intensity of parental drinking that may have a modeling effect for adolescents (consistent with the definition used in the previous review by Ryan et al., 2010). The rationale is that parents' problematic drinking patterns, such as abuse and dependency, are less modifiable by the parent themselves than frequency/intensity of drinking.

${ }^{\mathrm{b}}$ Parental discipline does not include variables that represent parental overcontrol, such as harsh discipline and excessive psychological control. 
Table 2. Criteria for risk of bias assessment

\begin{tabular}{|c|c|c|}
\hline Domain & Criterion & Description \\
\hline \multirow[t]{4}{*}{ Study participation } & A & $\begin{array}{l}\text { The sampling frame and recruitment were adequately described, } \\
\text { including the period and place of recruitment. }\end{array}$ \\
\hline & $\mathrm{B}$ & The inclusion and exclusion criteria were adequately described. \\
\hline & $\mathrm{C}$ & $\begin{array}{l}\text { The participation rate by eligible individuals at baseline was adequate, } \\
\text { and the sample size was sufficient. }{ }^{\text {a }}\end{array}$ \\
\hline & $\mathrm{D}$ & $\begin{array}{l}\text { The key characteristics of baseline study sample were adequately } \\
\text { described (at least for age and gender). }\end{array}$ \\
\hline \multirow[t]{2}{*}{ Study attrition } & $\mathrm{E}$ & $\begin{array}{l}\text { The response rate at time } 2 \text { was adequate, and there were no major } \\
\text { differences in key characteristics and outcomes between participants } \\
\text { who provided data at time } 2 \text { and those who did not. }{ }^{b}\end{array}$ \\
\hline & F & $\begin{array}{l}\text { The response rates at time } 3 \text { and/or at subsequent follow-up time points } \\
\text { were adequate, and there were no major differences in key } \\
\text { characteristics and outcomes between participants who provided data } \\
\text { and those who did not. }{ }^{\text {b }}\end{array}$ \\
\hline \multirow[t]{3}{*}{ Predictor measurement } & G & The description or definition of predictor variable was clear. \\
\hline & $\mathrm{H}$ & $\begin{array}{l}\text { Continuous variables were reported, or appropriate cut-points (i.e., not } \\
\text { data-dependent) were used. }\end{array}$ \\
\hline & I & $\begin{array}{l}\text { The predictor measurement and method were adequately valid and } \\
\text { reliable to limit misclassification bias. }\end{array}$ \\
\hline \multirow[t]{2}{*}{ Outcome measurement } & $\mathrm{J}$ & The description or definition of outcome variable was clear. \\
\hline & $\mathrm{K}$ & $\begin{array}{l}\text { The outcome measurement and method were adequately valid and } \\
\text { reliable to limit misclassification bias. }\end{array}$ \\
\hline \multirow[t]{2}{*}{ Analysis } & $\mathrm{L}$ & There was sufficient presentation of data. \\
\hline & M & There was no selective reporting of results. \\
\hline
\end{tabular}

${ }^{\text {a }}$ An adequate participation rate was defined as more than $80 \%$ of eligible individuals at baseline.

${ }^{\mathrm{b}}$ An adequate response rate was defined as more than $80 \%$ of baseline participants at time 2 , or $60-80 \%$ of baseline participants at time 3 and/or at subsequent follow-up time points. 
Table 3. Summary of findings for main analyses.

\begin{tabular}{|c|c|c|c|c|c|c|c|c|c|}
\hline Predictor & Outcome & $\begin{array}{l}\mathrm{N} \text { of } \\
\text { studies }\end{array}$ & $\begin{array}{l}N \text { of associations } \\
\text { in Stouffer's } p\end{array}$ & $\begin{array}{l}\text { Stouffer's } \\
p \text { value }\end{array}$ & $\begin{array}{l}\mathrm{N} \text { of associations } \\
\text { in meta-analysis }\end{array}$ & $\begin{array}{l}\mathrm{N} \text { of participants } \\
\text { in meta-analysis }\end{array}$ & $r(95 \% \mathrm{CI})$ & $I^{2}$ & $\begin{array}{l}\text { One-taile } \\
\text { for Egger }\end{array}$ \\
\hline \multicolumn{10}{|c|}{ Alcohol-specific parenting factors } \\
\hline \multirow{3}{*}{$\begin{array}{l}\text { Alcohol-specific } \\
\text { communication }\end{array}$} & Initiation of alcohol use & 10 & 10 & 0.207 & 2 & 661 & 0.124 & 94.696 & - \\
\hline & & & & & & & $(-0.137,0.369)$ & & \\
\hline & Levels of alcohol use/misuse & 4 & 5 & 0.259 & $2^{\mathrm{a}}$ & - & - & - & - \\
\hline \multirow{4}{*}{$\begin{array}{l}\text { Favorable attitudes } \\
\text { toward alcohol use }\end{array}$} & Initiation of alcohol use & 12 & 13 & $<0.0001$ & 5 & 1874 & 0.114 & $78.697 * *$ & 0.039 \\
\hline & & & & & & & $(0.033,0.193) * *$ & & \\
\hline & Levels of alcohol use/misuse & 17 & 24 & $<0.0001$ & 7 & 5911 & 0.182 & $62.327 *$ & 0.350 \\
\hline & & & & & & & $(0.139,0.224) * * *$ & & \\
\hline \multirow[t]{4}{*}{ Parental alcohol use } & Initiation of alcohol use & 20 & 20 & $<0.0001$ & 10 & 6456 & 0.116 & $93.170 * * *$ & 0.001 \\
\hline & & & & & & & $(0.049,0.182)^{* *}$ & & \\
\hline & Levels of alcohol use/misuse & 32 & 38 & $<0.0001$ & 12 & 7995 & 0.153 & $83.422 * * *$ & 0.395 \\
\hline & & & & & & & $(0.088,0.216) * * *$ & & \\
\hline \multirow[t]{4}{*}{ Provision of alcohol } & Initiation of alcohol use & 7 & 7 & $<0.0001$ & 2 & 2033 & 0.205 & 0.000 & - \\
\hline & & & & & & & $(0.162,0.248) * * *$ & & \\
\hline & Levels of alcohol use/misuse & 6 & 10 & $<0.0001$ & 4 & 3048 & 0.263 & 53.685 & - \\
\hline & & & & & & & $(0.210,0.314)^{* * * *}$ & & \\
\hline \multirow{3}{*}{$\begin{array}{l}\text { Rules about alcohol } \\
\text { use }\end{array}$} & Initiation of alcohol use & 7 & 8 & 0.0001 & 2 & 506 & -0.195 & 67.127 & - \\
\hline & & & & & & & $(-0.337,-0.044)^{*}$ & & \\
\hline & Levels of alcohol use/misuse & 6 & 6 & $<0.0001$ & 1 & - & - & - & - \\
\hline
\end{tabular}




\begin{tabular}{|c|c|c|c|c|c|c|c|c|c|}
\hline Predictor & Outcome & $\begin{array}{l}\mathrm{N} \text { of } \\
\text { studies }\end{array}$ & $\begin{array}{l}N \text { of associations } \\
\text { in Stouffer's } p\end{array}$ & $\begin{array}{l}\text { Stouffer's } \\
p \text { value }\end{array}$ & $\begin{array}{l}N \text { of associations } \\
\text { in meta-analysis }\end{array}$ & $\begin{array}{l}N \text { of participants } \\
\text { in meta-analysis }\end{array}$ & $r(95 \% \mathrm{CI})$ & $I^{2}$ & $\begin{array}{l}\text { One-tailed } p \text { value } \\
\text { for Egger's test }\end{array}$ \\
\hline \multicolumn{10}{|c|}{ Other general parenting factors } \\
\hline \multirow[t]{4}{*}{ Family conflict } & Initiation of alcohol use & 10 & 12 & $<0.0001$ & 8 & 9198 & 0.108 & $91.173 * * *$ & 0.001 \\
\hline & & & & & & & $(0.050,0.166) * * *$ & & \\
\hline & Levels of alcohol use/misuse & 13 & 18 & $<0.0001$ & 3 & 1259 & 0.112 & $81.175^{* *}$ & 0.442 \\
\hline & & & & & & & $(-0.026,0.245)$ & & \\
\hline \multirow{4}{*}{$\begin{array}{l}\text { General } \\
\text { communication }\end{array}$} & Initiation of alcohol use & 9 & 9 & $<0.0001$ & 2 & 1633 & -0.033 & 90.949 & - \\
\hline & & & & & & & $(-0.198,0.133)$ & & \\
\hline & Levels of alcohol use/misuse & 6 & 7 & 0.085 & 4 & 5051 & -0.029 & $91.176 * * *$ & 0.326 \\
\hline & & & & & & & $(-0.133,0.077)$ & & \\
\hline \multirow{4}{*}{$\begin{array}{l}\text { Parent-child } \\
\text { relationship quality }\end{array}$} & Initiation of alcohol use & 11 & 13 & $<0.0001$ & 6 & 7190 & -0.106 & $95.735 * * *$ & 0.042 \\
\hline & & & & & & & $(-0.190,-0.020)^{*}$ & & \\
\hline & Levels of alcohol use/misuse & 14 & 17 & $<0.0001$ & 8 & 22936 & -0.119 & $80.646 * * *$ & 0.209 \\
\hline & & & & & & & $(-0.156,-0.081)^{* * *}$ & & \\
\hline \multirow[t]{3}{*}{ Parental discipline } & Initiation of alcohol use & 19 & 19 & $<0.0001$ & 6 & 2107 & -0.069 & $73.912 * *$ & 0.106 \\
\hline & & & & & & & $(-0.134,-0.003)^{*}$ & & \\
\hline & Levels of alcohol use/misuse & 11 & 15 & $<0.0001$ & 1 & - & - & - & - \\
\hline \multirow[t]{4}{*}{ Parental involvement } & Initiation of alcohol use & 6 & 9 & $<0.0001$ & 5 & 11267 & -0.108 & $78.073^{* *}$ & 0.235 \\
\hline & & & & & & & $(-0.159,-0.057) * * *$ & & \\
\hline & Levels of alcohol use/misuse & 9 & 12 & $<0.0001$ & 5 & 19618 & -0.073 & $89.397 * * *$ & 0.047 \\
\hline & & & & & & & $(-0.126,-0.019) * *$ & & \\
\hline Parental monitoring & Initiation of alcohol use & 20 & 22 & $<0.0001$ & 7 & 8835 & -0.159 & $85.497 * * *$ & 0.121 \\
\hline
\end{tabular}




\begin{tabular}{|c|c|c|c|c|c|c|c|c|c|}
\hline Predictor & Outcome & $\begin{array}{l}\mathrm{N} \text { of } \\
\text { studies }\end{array}$ & $\begin{array}{l}N \text { of associations } \\
\text { in Stouffer's } p\end{array}$ & $\begin{array}{l}\text { Stouffer's } \\
p \text { value }\end{array}$ & $\begin{array}{l}N \text { of associations } \\
\text { in meta-analysis }\end{array}$ & $\begin{array}{l}N \text { of participants } \\
\text { in meta-analysis }\end{array}$ & $r(95 \% \mathrm{CI})$ & $I^{2}$ & $\begin{array}{l}\text { One-tailed } p \text { value } \\
\text { for Egger's test }\end{array}$ \\
\hline & & & & & & & $(-0.218,-0.098)^{* * * *}$ & & \\
\hline & Levels of alcohol use/misuse & 21 & 27 & $<0.0001$ & 12 & 15673 & -0.224 & $82.658 * * *$ & 0.432 \\
\hline & & & & & & & $(-0.267,-0.180)^{* * * *}$ & & \\
\hline \multirow[t]{4}{*}{ Parental support } & Initiation of alcohol use & 14 & 15 & $<0.0001$ & 8 & 6563 & -0.117 & $69.181^{* *}$ & 0.494 \\
\hline & & & & & & & $(-0.169,-0.065)^{* * * *}$ & & \\
\hline & Levels of alcohol use/misuse & 25 & 32 & $<0.0001$ & 7 & 7561 & -0.110 & $69.512^{* * *}$ & 0.413 \\
\hline & & & & & & & $(-0.154,-0.065) * * *$ & & \\
\hline
\end{tabular}

Notes: Associations were included in the main analyses based on the pre-specified selection hierarchy for each individual study: composite parental behaviors/parenting (first hierarchy), maternal behaviors/parenting (second), and paternal behaviors/parenting (third). A meta-analysis was not conducted when there were fewer than two independent studies available. Significant pooled effect sizes are presented in bold. $\mathrm{N}=$ Total number; $r=$ pooled effect size (correlation coefficient); $\mathrm{CI}=$ confidence interval; $I^{2}=$ indicator of heterogeneity in percentages. $*^{*} p<0.05 ; * * p<0.01 ; * * * p<0.001$.

${ }^{a}$ A meta-analysis was not conducted since the two associations were extracted from the same study. 
Table 4. Results of supplementary meta-analyses for mothers and fathers.

\begin{tabular}{|c|c|c|c|c|c|c|c|c|c|}
\hline \multirow{2}{*}{ Predictor } & \multirow{2}{*}{ Outcome } & \multicolumn{4}{|c|}{ Analysis for mothers } & \multicolumn{4}{|c|}{ Analysis for fathers } \\
\hline & & $\begin{array}{l}\mathrm{N} \text { of } \\
\text { associations }\end{array}$ & $\begin{array}{l}\mathrm{N} \text { of } \\
\text { participants }\end{array}$ & $r(95 \% \mathrm{CI})$ & $I^{2}$ & $\begin{array}{l}\mathbf{N} \text { of } \\
\text { associations }\end{array}$ & $\begin{array}{l}\mathbf{N} \text { of } \\
\text { participants }\end{array}$ & $r(95 \% \mathrm{CI})$ & $I^{2}$ \\
\hline \multicolumn{10}{|c|}{ Alcohol-specific parenting factors } \\
\hline \multirow{3}{*}{$\begin{array}{l}\text { Alcohol-specific } \\
\text { communication }\end{array}$} & Initiation of alcohol use & 2 & 661 & 0.125 & 94.602 & 2 & 597 & 0.065 & 72.353 \\
\hline & & & & $(-0.135,0.369)$ & & & & $(-0.062,0.191)$ & \\
\hline & Levels of alcohol use/misuse & $2^{\mathrm{a}}$ & - & - & - & $2^{\mathrm{a}}$ & - & - & - \\
\hline \multirow{4}{*}{$\begin{array}{l}\text { Favorable attitudes } \\
\text { toward alcohol use }\end{array}$} & Initiation of alcohol use & 4 & 1661 & 0.093 & $79.863 * *$ & 4 & 1597 & 0.058 & 20.782 \\
\hline & & & & $(-0.007,0.191)$ & & & & $(0.015,0.101)^{* *}$ & \\
\hline & Levels of alcohol use/misuse & 4 & 2210 & 0.175 & 0.000 & 4 & 2210 & 0.167 & 5.289 \\
\hline & & & & $(0.134,0.215)^{* * *}$ & & & & $(0.125,0.209)^{* * *}$ & \\
\hline \multirow[t]{4}{*}{ Parental alcohol use } & Initiation of alcohol use & 5 & 2639 & 0.081 & $88.671 * * *$ & 4 & 1783 & 0.114 & $90.561 * * *$ \\
\hline & & & & $(-0.001,0.161)$ & & & & $(0.005,0.220)^{*}$ & \\
\hline & Levels of alcohol use/misuse & 8 & 5960 & 0.159 & $68.571 * *$ & 5 & 2782 & 0.158 & $72.973 * *$ \\
\hline & & & & $(0.101,0.216)^{* * * *}$ & & & & $(0.085,0.231)^{* * * *}$ & \\
\hline \multirow[t]{2}{*}{ Provision of alcohol } & Initiation of alcohol use & 0 & - & - & - & 0 & - & - & - \\
\hline & Levels of alcohol use/misuse & 0 & - & - & - & 0 & - & - & - \\
\hline \multirow{2}{*}{$\begin{array}{l}\text { Rules about alcohol } \\
\text { use }\end{array}$} & Initiation of alcohol use & 1 & - & - & - & 1 & - & - & - \\
\hline & Levels of alcohol use/misuse & 0 & - & - & - & 0 & - & - & - \\
\hline
\end{tabular}




\begin{tabular}{|c|c|c|c|c|c|c|c|c|c|}
\hline \multirow{2}{*}{ Predictor } & \multirow{2}{*}{ Outcome } & \multicolumn{4}{|c|}{ Analysis for mothers } & \multicolumn{4}{|c|}{ Analysis for fathers } \\
\hline & & $\begin{array}{l}\mathrm{N} \text { of } \\
\text { associations }\end{array}$ & $\begin{array}{l}\mathrm{N} \text { of } \\
\text { participants }\end{array}$ & $r(95 \% \mathrm{CI})$ & $I^{2}$ & $\begin{array}{l}\mathrm{N} \text { of } \\
\text { associations }\end{array}$ & $\begin{array}{l}\mathrm{N} \text { of } \\
\text { participants }\end{array}$ & $r(95 \% \mathrm{CI})$ & $I^{2}$ \\
\hline \multicolumn{10}{|c|}{ Other general parenting factors } \\
\hline \multirow[t]{2}{*}{ Family conflict } & Initiation of alcohol use & 1 & - & - & - & 1 & - & - & - \\
\hline & Levels of alcohol use/misuse & 1 & - & - & - & 1 & - & - & - \\
\hline \multirow{3}{*}{$\begin{array}{l}\text { General } \\
\text { communication }\end{array}$} & Initiation of alcohol use & 0 & - & - & - & 1 & - & - & - \\
\hline & Levels of alcohol use/misuse & 4 & 5051 & -0.029 & $91.176 * * *$ & 4 & 5051 & -0.053 & $76.769 * *$ \\
\hline & & & & $(-0.133,0.077)$ & & & & $(-0.119,0.014)$ & \\
\hline \multirow{4}{*}{$\begin{array}{l}\text { Parent-child } \\
\text { relationship quality }\end{array}$} & Initiation of alcohol use & 3 & 2524 & -0.036 & $72.808^{*}$ & 2 & 1405 & -0.032 & 71.762 \\
\hline & & & & $(-0.085,0.014)$ & & & & $(-0.089,0.026)$ & \\
\hline & Levels of alcohol use/misuse & 2 & 4860 & $-\mathbf{- 0 . 1 3 3}$ & 0.000 & 2 & 4860 & -0.172 & 0.000 \\
\hline & & & & $(-0.160,-0.105)^{* * * *}$ & & & & $(-0.199,-0.144) * * *$ & \\
\hline \multirow[t]{3}{*}{ Parental discipline } & Initiation of alcohol use & 3 & 737 & -0.126 & 54.609 & 3 & 673 & -0.064 & 39.536 \\
\hline & & & & $(-0.233,-0.016)^{*}$ & & & & $(-0.163,0.035)$ & \\
\hline & Levels of alcohol use/misuse & 0 & - & - & - & 0 & - & - & - \\
\hline \multirow[t]{2}{*}{ Parental involvement } & Initiation of alcohol use & 0 & - & - & - & 1 & - & - & - \\
\hline & Levels of alcohol use/misuse & 1 & - & - & - & 1 & - & - & - \\
\hline \multirow[t]{2}{*}{ Parental monitoring } & Initiation of alcohol use & 0 & - & - & - & 0 & - & - & - \\
\hline & Levels of alcohol use/misuse & 1 & - & - & - & 1 & - & - & - \\
\hline Parental support & Initiation of alcohol use & 4 & 857 & -0.163 & $79.215^{* *}$ & 4 & 793 & -0.095 & 57.566 \\
\hline
\end{tabular}




\begin{tabular}{|c|c|c|c|c|c|c|c|c|c|}
\hline \multirow{2}{*}{ Predictor } & \multirow{2}{*}{ Outcome } & \multicolumn{4}{|c|}{ Analysis for mothers } & \multicolumn{4}{|c|}{ Analysis for fathers } \\
\hline & & $\begin{array}{l}\mathrm{N} \text { of } \\
\text { associations }\end{array}$ & $\begin{array}{l}\text { Nof of } \\
\text { participants }\end{array}$ & $r(95 \% \mathrm{CI})$ & $I^{2}$ & $\begin{array}{l}\mathrm{N} \text { of } \\
\text { associations }\end{array}$ & $\begin{array}{l}\text { of of } \\
\text { participants }\end{array}$ & $r(95 \% \mathrm{CI})$ & $I^{2}$ \\
\hline & \multirow{3}{*}{ Levels of alcohol use/misuse } & \multirow{3}{*}{3} & \multirow{3}{*}{4534} & $(-0.307,-0.012)^{*}$ & \multirow{3}{*}{62.959} & \multirow{3}{*}{3} & \multirow{3}{*}{4534} & $(-0.203,0.016)$ & \multirow{3}{*}{49.404} \\
\hline & & & & -0.147 & & & & -0.131 & \\
\hline & & & & $(-0.202,-0.091)^{* * * *}$ & & & & $(-0.177,-0.084) * * * *$ & \\
\hline
\end{tabular}

Notes: A meta-analysis was not conducted when there were fewer than two independent studies available. Significant pooled effect sizes are presented in bold. $\mathrm{N}=$ Total number; $r=$ pooled effect size (correlation coefficient); $\mathrm{CI}=$ confidence interval; $P^{2}=$ indicator of heterogeneity in percentages. ${ }^{*} p<0.05 ; * * p<0.01 ; * * *<0.001$.

${ }^{a}$ A meta-analysis was not conducted since the two associations were extracted from the same study. 
